\title{
侧链调控在有机光电高分子材料中的应用研究进展
}

\author{
张超孙莹戴斌张雪勤 \\ 杨 洪林保平* 郭玲香 \\ (东南大学化学化工学院 南京 211189)
}

\begin{abstract}
摘要 有机光电聚合物材料是由共轭主链与柔性侧链组成, 具有制备简单、成本低廉、重量轻及可制成柔性器件等突 出优点, 近年来成为国内外研究的前沿和热点. 目前对聚合物材料主链结构的优化研究较多，基于侧链调控的报道则 相对较少. 对主链共轭高分子光电材料侧链调控的目的主要是改善材料的溶解性能, 然而, 侧链修饰的影响远非如此. 研究发现, 侧链修饰后聚合物材料的吸收光谱、能级分布、载流子迁移率、器件共混膜形貌以及界面形态等光电性能 方面可发生不同程度的变化. 此类研究成果无疑将对设计与优化有机聚合物材料分子结构、活性层形貌与界面形态以 及器件制备方法和加工工艺具有指导意义. 同时, 随着器件制备方法的不断改进以及加工工艺的不断发展, 为进一步 改善器件效率, 提高纳米活性材料的形貌调控与界面形态优化能力, 基于有机光电高分子材料侧链调控的研究价值日 益突显. 因此, 结合高分子光电材料应用领域的研究现状, 以聚合物分子侧链结构优化设计为出发点, 基于侧链调控 为主题, 以不同的修饰侧链为研究对象, 对光电高分子材料侧链调控中拟解决的问题及本实验室的相关工作做主要综 述.
\end{abstract}

关键词 侧链调控; 光电性能; 聚合物太阳能电池; 膜形貌

\section{Resent Progress in Side-Chain Engineering of Organic Photovoltaic Conjugated Polymer}

\author{
Zhang, Chao Sun, Ying Dai, Bin Zhang, Xueqin \\ Yang, Hong Lin, Baoping* Guo, Lingxiang \\ (Department of Chemistry and Chemical Engineering, Southeast University, Nanjing 211189)
}

\begin{abstract}
Photovoltaic conjugated polymer materials are composed of $\pi$-conjugated backbone and flexible side chains. Their potential advantages of cost-effective production, fabrication on flexible and light weight substrates by roll-to-roll solution processing, capability to be fabricated into flexible devices and reduced environment impact have made them receive considerable attention in both academia and industry. In recent years, most of the interest has been directed into the optimization of main chain, while research on side-chain engineering of polymer is relatively few. Initially, side chains have been primarily utilized as solubilizing groups in organic photovoltaic conjugated polymers. However, roles which side chains play are far beyond. Accordingly, it has been found that side chains of organic conjugated polymer have a different impact on polymer absorption, emission, energy level, carrier mobility, nanoscale morphology and interfacial contact. Certainly enough, these findings have profound guiding significance on molecular structure design and optimization, blend microstructure, interfacial morphology, preparation methods and processing technology of device and so on. Moreover, based on ameliorative preparation and processing technology of device, side-chain engineering is deserved to be deeply researched due to their remarkably improved ability to regulate and control blend microstructure, interfacial morphology so as to obtain ideal photovoltaic properties and device performance, the ultimate goal. Thus, as for resent progress in application, in this perspective article, we will present a review on side chain engineering and assess different side chain on the basis of molecular structure design and optimization, as well as summarize relevant issues to be solved and related work in our laboratory.

Keywords side chain engineering; photovoltaic properties; polymer solar cells; nanoscale morphology
\end{abstract}

\footnotetext{
*E-mail: 1bp@seu.edu.cn

Received March 27, 2014; revised April 26, 2014; published online May 23, 2014 (Nos. BK20130619, BK20130617).

国家自然科学基金(Nos. 21304018, 21374016)和江苏省自然科学基金(Nos. BK20130619, BK20130617)资助项目.
}

Project supported by the National Natural Science Foundation of China (Nos. 21304018, 21374016) and the Jiangsu Provincial Natural Science Foundation 
有机共轭聚合物材料主要由共轭主链和柔性侧链 构成 ${ }^{[1,2]}$, 具有结构多样化、可调控性能佳、制备工艺简 单、可通过溶液处理加工制成柔性器件等显著优点 ${ }^{[35]}$, 此外, 共轭聚合物材料良好的光吸收性能、优异的载流 子传输性能以及良好的热稳定性能使其在有机太阳能 电池 ${ }^{[6 \sim 8]}$ 、有机场效应晶体管 ${ }^{[9 \sim 11]}$ 以及有机发光器 件 ${ }^{[12 \sim 15]}$ 等领域中得到广泛应用. 因此, 新型有机共轭聚 合物的设计与制备受到学界和产业界的极大关注. 随着 对共轭聚合物材料分子结构及器件制备研究的不断深 入, 共轭聚合物材料的光电性能得到了显著提高. 基于 共轭聚合物材料本体异质结聚合物太阳能电池(PSC)的 光电转换效率已经超过 $8 \%{ }^{[16 ~ 20]}$. 基于共轭聚合物的有 机单晶场效应管(OFETs)与非晶硅有源层的场效应管相 比也显示出了同样优异的性能, 其载流子迁移率也达到 了 $10 \mathrm{~cm}^{2} \cdot \mathrm{V}^{-1} \cdot \mathrm{s}^{-1[21 \sim 25]}$.

众所周知, 通过对光电高分子材料加工工艺参数和 器件制备方法的调控, 可以在一定程度上提高器件的光 电性能, 然而整个优化过程繁琐复杂, 影响因素众多, 可重复性差, 不利于光电器件的大规模生产与应用 ${ }^{[23]}$, 而聚合物分子的结构设计与调控更为简单有效. 由于聚 合物材料的 $\pi$ 共轭主链是影响材料光电性能的重要因 素 ${ }^{[26 ~ 28]}$, 故目前对聚合物材料主链结构的优化研究较 多, 基于侧链调控的报道则相对较少. 但近些年来, 不 少创造性的研究报道侧链修饰亦可有效改善聚合物材 料的载流子迁移性能以及器件的共混膜形貌和界面形 态, 最终改善材料的光电特性 ${ }^{[29-32]}$. 故而, 有机光电材 料中的侧链调控研究为学者们提供了新的思路, 对设计 和优化有机聚合物材料分子结构、活性层形貌、界面形 态以及器件制备方法和加工工艺亦具有指导意义 ${ }^{[33 \sim 38]}$.

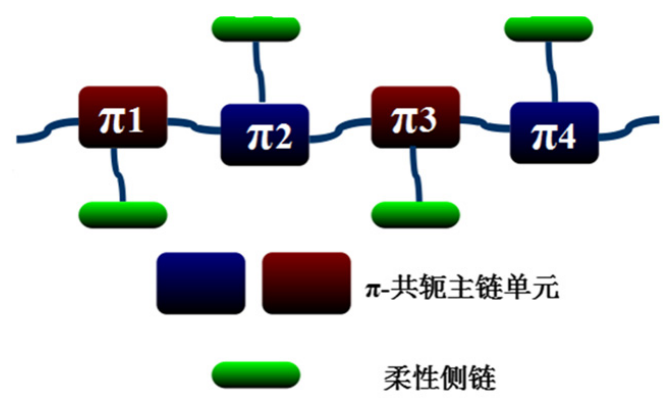

图 1 共轭聚合物的结构示意图

Figure 1 Structural schematic diagram of conjugated polymer

本文结合光电高分子材料应用领域的研究近况, 以 聚合物材料分子结构设计为出发点, 侧链调控为主题, 以不同的修饰侧链为研究对象, 基于近年来代表性的报 道与研究以系统阐述聚合物材料侧链修饰对其吸收光 谱、能级分布、载流子迁移率、器件共混膜形貌以及界 面形态等光电性能方面的影响.

\section{1 光电功能高分子侧链修饰研究}

根据现有的相关研究，应用于有机光电高分子材料 的侧链按结构可分为: 烷基型、混合型、水/醇溶型、氟 取代型以及末端功能化基团型侧链等.

\section{1 烷基型侧链}

烷基侧链在制备过程中简单易行，对高分子材料修 饰的可行性强, 并且可在不破坏主链共轭强度的前提 下, 有效改善材料在溶液中的溶解性能 ${ }^{[39 ~ 42]}$, 提高材料 在溶液中的加工处理性能.

鉴于工艺制备条件的限制, 偶数碳烷基比奇数碳烷 基侧链更易获得，诸如己基、辛基、十二烷基和十八烷 基作为长链烷基均已广泛应用于给受体型共轭聚合物 体系中 ${ }^{[40 \sim 44]}$. 文献报道 ${ }^{[41]}$, 有机小分子光电材料中, 侧 链烷基碳数的奇偶性对光电性能亦有所影响, 而在聚合 物体系中尚未发现类似现象.此外，从结构上看，烷基 侧链又可分为直链型和支链型. 支链型烷基较直链烷基 具备更加出色的溶解性，但基于支链烷基的不利位阻, 直链烷基侧链更利于诱导链间的相互作用.

研究发现，长链烷基虽可有效改善聚合物材料的溶 解性能，但却不利于诱导高分子链的聚集，导致载流子 迁移率下降, 进而不利于聚合物材料光电性能的提 高 ${ }^{[42,43]}$. 为系统和深入的探究侧链烷基对聚合物光电性 能影响的内在机制, Deng 等 ${ }^{[4]}$ 以二噻吩并咔唑(DTC)为 给体单元，二酮吡咯并吡咯(DPP)为受体单元，通过对 DTC 给体单元链接不同烷基侧链的方式, 制备出新型 的给受体型共轭聚合物 $\mathbf{P 1}\left(M_{\mathrm{n}}=16800, \mathrm{PDI}=2.92\right), \mathbf{P 2}$ $\left(M_{\mathrm{n}}=46600, \mathrm{PDI}=2.31\right), \mathbf{P 3}\left(M_{\mathrm{n}}=27900, \mathrm{PDI}=2.63\right)$ 和 P4 $\left(M_{\mathrm{n}}=16800, \mathrm{PDI}=2.92\right)$. 研究发现, 在此聚合物体 系中, 不同的烷基对聚合物的 HOMO 与 LUMO 能级的 影响并不大，但对聚合物的光捕获能力、载流子传输性 以及材料与 PC71BM 的共混性等均有显著影响, 含有直 链烷基的聚合物 $\mathbf{P 1}$ 与 $\mathbf{P 2}$ 光电性能更为优越. 原因在于, P3，P4 与受体材料在二氯苯 $(o-D C B)$ 溶液中的溶解性差 异变大, 致使 PC71BM 发生较为严重的聚集现象, 使得 光敏活性层中相分离尺寸过大，载流子复合几率增加， 导致能量转换效率(PCE)分别只有 $0.97 \%$ 和 $1.1 \%$. 其中, $\mathbf{P 1}$ 的光电转换效率最优, 与 PC71BM 以 $1: 3$ 的比例共 混制备本体异质结电池器件光电器件电流密度达到 $13.4 \mathrm{~mA} / \mathrm{cm}^{2}$, 开路电压 $V_{\mathrm{oc}}=0.72 \mathrm{~V}, \mathrm{FF}=62 \%$, 器件转 化效率达到 $5.7 \%$.

2012年 Ma 等 ${ }^{[45]}$ 报道的全聚合物型本体异质结光伏 器件则是基于苯并二噻吩(BDT)为给体单元, 异靛蓝为 受体单元的新型共轭聚合物给体材料, 并对噻吩 $\pi$ 桥进 一步烷基化修饰制备出聚合物 P6 $\left(M_{\mathrm{n}}=90000, \mathrm{PDI}=\right.$ 

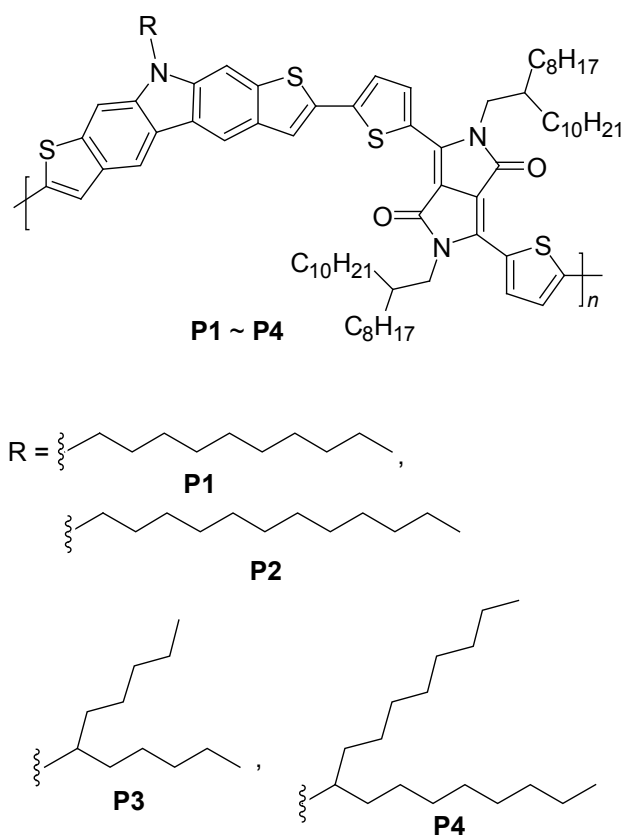

2.7), 研究发现, 经辛基修饰的 P6, 在溶液和薄膜状态 下, 吸收光谱变化不大, 轨道能级与能带间隙也几乎相 同, 然而光电性能有了很大的变化. 通过密度泛函理论 (DFT)计算发现, 烷基修饰的 P6 存在的二面角(23.1 ${ }^{\circ}$ 远 大于聚合物 P5 $\left(10.6^{\circ}\right)$, 这就使得由于侧链烷基的位阻 效应, P6 的主链与相邻噻吩环的共平面性变差, 共轭程 度减弱, 从而不利于聚合物链间的 $\pi-\pi$ 相互作用, 最终 导致 P6 与 PC61BM 以 1:2 的比例共混制备的本体异 质结电池器件光电转化效率从 $3.84 \%\left(\mathbf{P 5}: J_{\mathrm{sc}}=7.46 \mathrm{~mA} /\right.$ $\mathrm{cm}^{2}, V_{\mathrm{oc}}=0.78 \mathrm{~V}, \mathrm{FF}=66 \%$ )减少至 $1.39 \%\left(\mathbf{P 6}: J_{\mathrm{sc}}=3.15\right.$ $\mathrm{mA} / \mathrm{cm}^{2}, V_{\mathrm{oc}}=0.73 \mathrm{~V}, \mathrm{FF}=60 \%$ ).

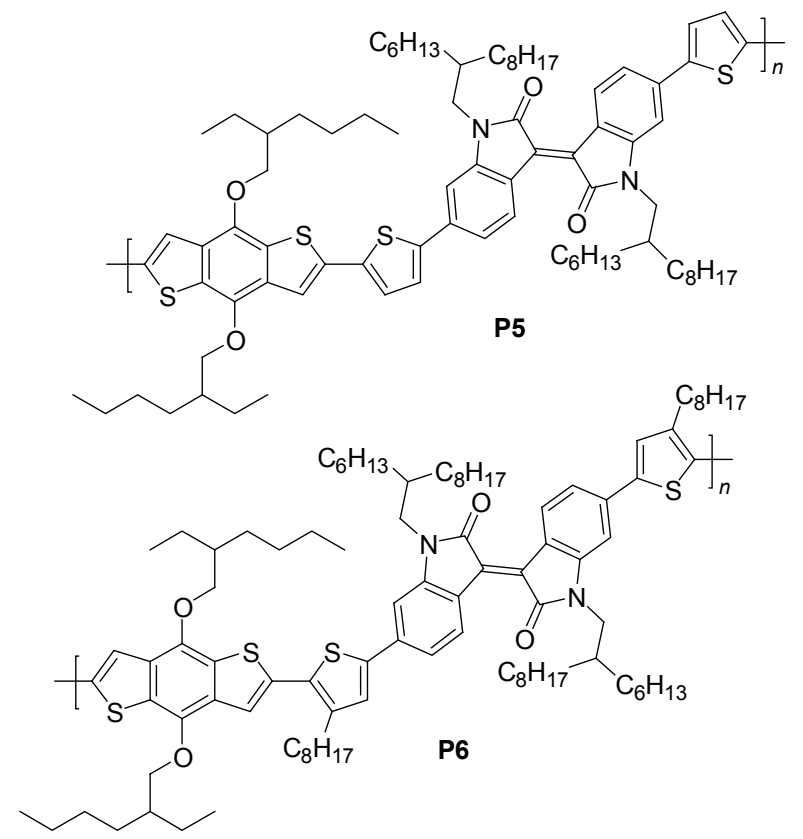

随后, 2013 年 $\mathrm{Li}$ 等 ${ }^{[46]}$ 基于环戊二烯双噻吩(CPT)制 备了具有不同烷基侧链的聚合物 $\mathbf{P 7}\left(M_{\mathrm{n}}=25300, \mathrm{PDI}=\right.$ $1.58)$ 与 $\mathbf{P 8}\left(M_{\mathrm{n}}=24600, \mathrm{PDI}=1.47\right)$. 结构上不难看出, 3,7-二甲基辛基修饰的 P8 具备更大的侧链位阻. 虽然具 有相同的共轭主链, 但 P7 的起始氧化电位比相对应的 $\mathbf{P 8}$ 增加了约 $100 \mathrm{mV}$. 结合 XRD 测试结果, P7 具备更小 的层间堆叠距离, 作者推测, 可能由于 3,7-二甲基辛基 的较大位阻, 弱化了聚合物分子链间的相互作用. 此外, 还发现 P8 中具有更少的有序结构组织, 原因在于聚合 物 P7 有两个明显的衍射峰, 而 P8 只有一个. 这一推论 在聚合物材料紫外吸收图谱中也得到了证明, 聚合物 P7 在长波段有明显的肩峰, 而 P8 却没有. 分子链间较 大的距离以及较弱的作用力使得聚合物材料的聚集性 能降低, 更弱的链间作用及更大的相互作用距离, 导致 了不佳的聚集性能, 空穴迁移率相比较于 P7 的 $1.4 \times$ $10^{-2} \mathrm{~cm}^{2} \cdot \mathrm{V}^{-1} \cdot \mathrm{s}^{-1}$ 减少至 $3.0 \times 10^{-4} \mathrm{~cm}^{2} \cdot \mathrm{V}^{-1} \cdot \mathrm{s}^{-1}$. 载流子 迁移率的下降也使得器件的短路电流从 $14.5 \mathrm{~mA} / \mathrm{cm}^{2}$ 锐 减至 $4.76 \mathrm{~mA} / \mathrm{cm}^{2}$, 同等条件制备的本体异质结电池器 件也从 $5.48 \%$ 降至 $1.29 \%$.
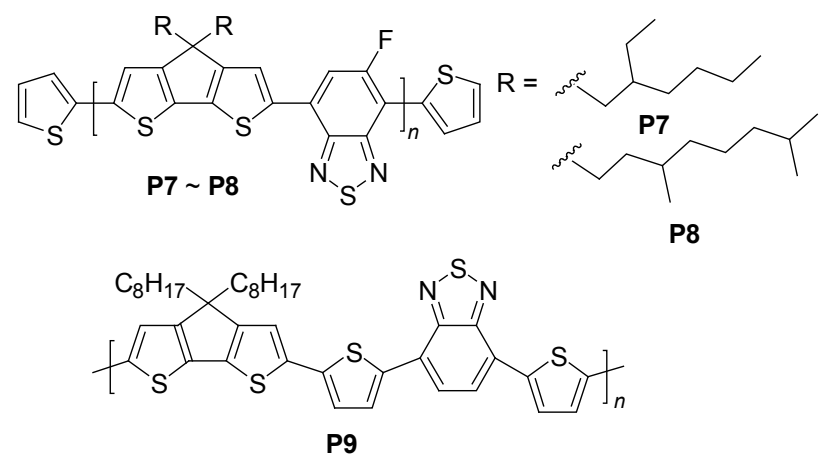

众所周知，烷基侧链位置的不同，会导致其在空间 取向上有所差异 ${ }^{[47]}$, 故而, 对烷基侧链位置的选择值得 深入探讨和系统研究. Lee 等 ${ }^{[48]}$ 报道了 P10 $\left(M_{\mathrm{n}}=9000\right.$, $\mathrm{PDI}=1.3)$ 和 $\mathbf{P 1 1}\left(M_{\mathrm{n}}=12000, \mathrm{PDI}=2.0\right)$ 两种新型的共 轭聚合物, 发现侧链烷基所处的位置对聚合物的光伏性 能影响很大. 由于烷基侧链的不利位阻, P10 与 P11 紫 外吸收光谱较无烷基修饰的聚合物 P9 都发生不同程度 的蓝移, 其中P10 蓝移 $40 \mathrm{~nm}, \mathbf{P 1 1}$ 蓝移多达 $146 \mathrm{~nm}$, 聚 合物能带间隙也由 $1.50 \mathrm{eV}$ 分别增加至 1.62 和 $1.83 \mathrm{eV}$. 此外, 制备有机场效应晶体管(OFET)器件, 对聚合物材 料的载流子迁移率进行测试, 结果发现 P10 空穴迁移率 与 $\mathbf{P 9}$ 相比虽然减小, 但二者相差并不大, 分别为 $\mathbf{P 9}$ : $\mu_{\mathrm{h}}=4.9 \times 10^{-3} \mathrm{~cm}^{2} \cdot \mathrm{V}^{-1} \cdot \mathrm{s}^{-1} ; \mathbf{P 1 0}: \mu_{\mathrm{h}}=1.8 \times 10^{-3} \mathrm{~cm}^{2}$ • $\mathrm{V}^{-1} \cdot \mathrm{s}^{-1}$; 而 P11 的空穴迁移率却明显锐减, 只有 $\mu_{\mathrm{h}}=$ $3.8 \times 10^{-6} \mathrm{~cm}^{2} \cdot \mathrm{V}^{-1} \cdot \mathrm{s}^{-1}$, 以上现象可归因于: (1) P3HT 等 噻吩类聚合物链中在相邻噻吩之间引入烷基侧链对其 
二面角改变较小, 对分子的平面性影响亦不大, 可以达 到溶解性和分子平面性的平衡. P10 中烷基侧链位于噻 吩与噻吩之间, 对聚合物主链的平面性影响较弱, 电子 依然能够很好的离域, 分子内电荷依然可以有效转移, 因此聚合物 P10 与聚合物 P9 的空穴迁移率相差不大. (2)较 P10 而言, P11 中噻吩 $\pi$ 桥上的烷基链则位于噻吩 环与苯环之间, 产生了较大的二面角, 破坏了分子的平 面性, 导致主链的有效共轭程度降低, 给体单元的电子 无法通过噻吩 $\pi$ 桥传递到受体单元, 制约了分子内电荷 转移, 此推论从材料的光物理测试中也得到了很好的印 证, P11 的紫外吸收光谱发生严重蓝移. 与此同时, 主链 较差的平面性还导致聚合物链间 $\pi-\pi$ 相互作用减弱, 聚 集态堆积无序性增加, 最终造成其空穴迁移率最差.

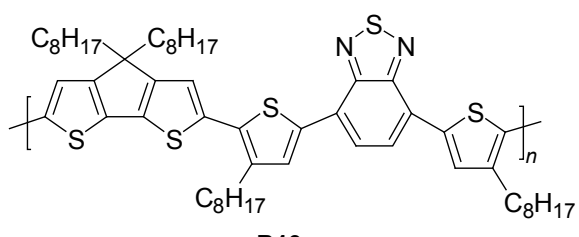

P10

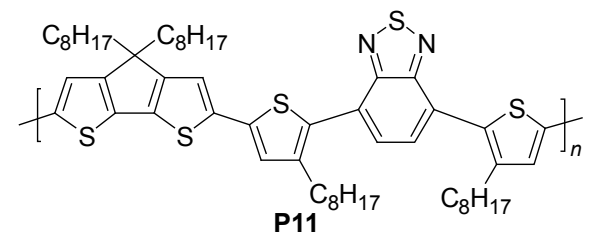

为了弥补支链烷基位阻效应的缺陷，近年来，对支 链烷基侧链的改性研究亦备受关注 ${ }^{[49,50]}$. 2013 年 $\mathrm{Kim}$ 等 ${ }^{[51]}$ 基于 DPP 制备了聚合物材料 P12 $\left(M_{\mathrm{n}}=31900\right.$, PDI $=1.7)$, 其结构特征在于, 在受体单元 DPP 两侧各有 一支化烷基长链, 而后通过延长支链中心碳与氮原子之 间直链烷基的长度，合成了具有更佳溶解性的 P13 $\left(M_{\mathrm{n}}=33530, \mathrm{PDI}=1.78\right)$. 结果发现, 溶解性能的增强, 有效提高了聚合物分子的自组装性能. 此外, P13 的 $\mathrm{HOMO}$ 能级亦有所改变, 从 $-5.31 \mathrm{eV}$ 增加至 $-5.25 \mathrm{eV}$, 此现象在紫外吸收图谱中也得到了很好的验证. 与 $\mathbf{P 1 2}$ 相比, P13 在溶液和薄膜状态下均红移了 $20 \mathrm{~nm}, \mathbf{P 1 3}$ 的 FET 测试亦显示出更强的载流子迁移性能, 从 $0.2 \mathrm{~cm}^{2}$ $\mathrm{V}^{-1} \cdot \mathrm{s}^{-1}$ 增加至 $5.1 \mathrm{~cm}^{2} \cdot \mathrm{V}^{-1} \cdot \mathrm{s}^{-1}$. 作者推测, 随着线型烷 基中亚甲基长度的延展与拓宽, 侧链烷基静态位阻效应 得到改善, 链间 $\pi-\pi$ 相互作用距离减小, 利于诱导更好 的分子间 $\pi-\pi$ 堆积以及快速有效的电荷传输, 避免电荷 复合损失. 随后他们 ${ }^{[51]}$ 在研究聚合物 P14 $\left(M_{\mathrm{n}}=33369\right.$, $\mathrm{PDI}=1.82)$ 与 $\mathrm{P} 15\left(M_{\mathrm{n}}=35826, \mathrm{PDI}=1.62\right)$ 时也发现类 似的现象, P15 的载流子迁移率从 $0.47 \mathrm{~cm}^{2} \cdot \mathrm{V}^{-1} \cdot \mathrm{s}^{-1}$ 增加 至 $5.8 \mathrm{~cm}^{2} \cdot \mathrm{V}^{-1} \cdot \mathrm{s}^{-1}$, 并且发现 $200{ }^{\circ} \mathrm{C}$ 热处理后的薄膜载 流子迁移率可得到显著改善, 在最优的工艺参数和器件
结构下, P13 与 P15 的载流子迁移率更高达 10.54 和 $12.04 \mathrm{~cm}^{2} \cdot \mathrm{V}^{-1} \cdot \mathrm{s}^{-1}$.

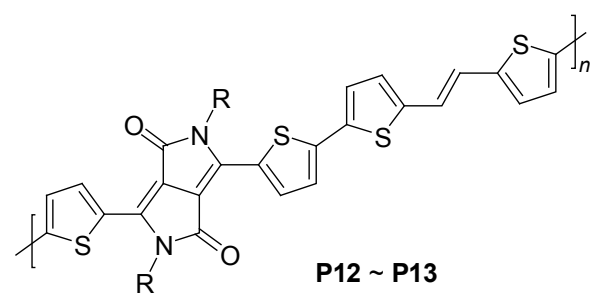

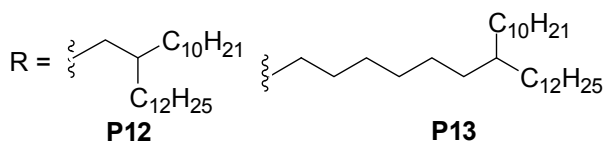<smiles>[R]N1C(=O)C2=C(c3ccc(-c4ccc(/C=C/c5ccc(C)o5)s4)s3)N([R])C(=O)C2=C1c1ccc(C)s1</smiles>

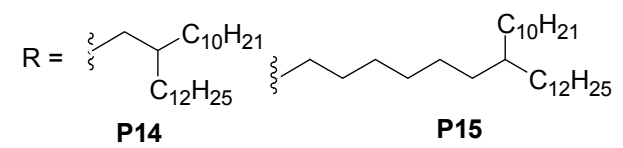

综上所述，烷基侧链的位置、长度以及位阻均可影 响聚合物链间的 $\pi-\pi$ 相互作用，进而制约聚合物材料的 电荷传输及器件性能. 在 OFET 中, 链间 $\pi-\pi$ 相互作用 的距离减小, 利于诱导更为理想的分子间 $\pi-\pi$ 堆积以及 快速有效的电荷传输; 然而, 对于 OPV 而言, 却不利于 开路电压 $V_{\mathrm{oc}}$ 的提高. 于此同时, 由于纳米活性层膜形 貌、聚合物自身纯度及分子量的协同影响, 在实际的研 究过程中, 我们依然很难预测烷基侧链对于聚合物材料 吸收光谱、能级分布及载流子迁移率等方面的影响.

\section{2 混合型侧链}

\section{2 .1 烷氧基型侧链}

烷氧基侧链的引入可有效改善材料的溶解性和成 膜性，使聚合物材料更易在溶液体系中操作加工 ${ }^{[52,54]}$, 此外, 其优势还在于可降低材料的能带隙, 延长其吸收 起始波长, 且烷氧基侧链更易在芳香环上引入. 但其弊 端也是显而易见的，烷氧基会有效增加聚合物的 HOMO 能级, 鉴于材料的 HOMO 能级与电子受体的 LUMO 能级之差, 直接影响器件的开路电压 $V_{\mathrm{oc}}{ }^{[55 \sim 57]}$, 故相应的, 电子给体的 HOMO 能级越高, 越不利于器件 开路电压的改善. 因此，基于烷氧基对聚合物侧链进行 结构调控研究, 需要统筹能带隙和器件开路电压两个相 互矛盾的因素.

$\mathrm{Hou}$ 等 ${ }^{[58]}$ 利用简单的苯并二噻吩(BDT)和苯并噻二 唑(BT)构筑窄带隙线型聚合物 P16 $\left(M_{\mathrm{n}}=23200, \mathrm{PDI}=\right.$ 
1.3) 和 P17 $\left(M_{\mathrm{n}}=19200, \mathrm{PDI}=1.2\right)$. 两聚合物 $\mathbf{P 1 6}$ 和 $\mathbf{P 1 7}$ 的不同之处在于噻吩侧链处链接的分别为烷基和烷氧 基, 并探讨已基与己基氧基侧链对聚合物材料的光谱、 电化学、能级结构的影响差异. 研究发现, P17 的主链获 得了更强的共平面性及更优良的载流子迁移率. 经证 实, P17 的紫外吸收光谱的确得到了一定的拓宽, 相比 P16, 聚合物 P17 在溶液与薄膜状态下分别红移了 49 和 $94 \mathrm{~nm}$. 这主要归因于烷氧基较强的推电子特性, 使得 $\mathrm{HOMO}$ 轨道能级在此基础上增加了 $0.4 \mathrm{eV}$, 能带间隙也 从 $1.85 \mathrm{eV}$ 减小至 $1.55 \mathrm{eV}$, 但由于 $\mathrm{HOMO}$ 能级的提高, 导致相应器件的开路电压从 $0.84 \mathrm{~V}$ 减小至 $0.58 \mathrm{~V}$. 制备 的本体异质结电池器件, 同等条件下 P16 与 $\mathbf{P 1 7}$ 光电转 化效率分别为 $0.85 \%$ 和 $1.08 \%$.

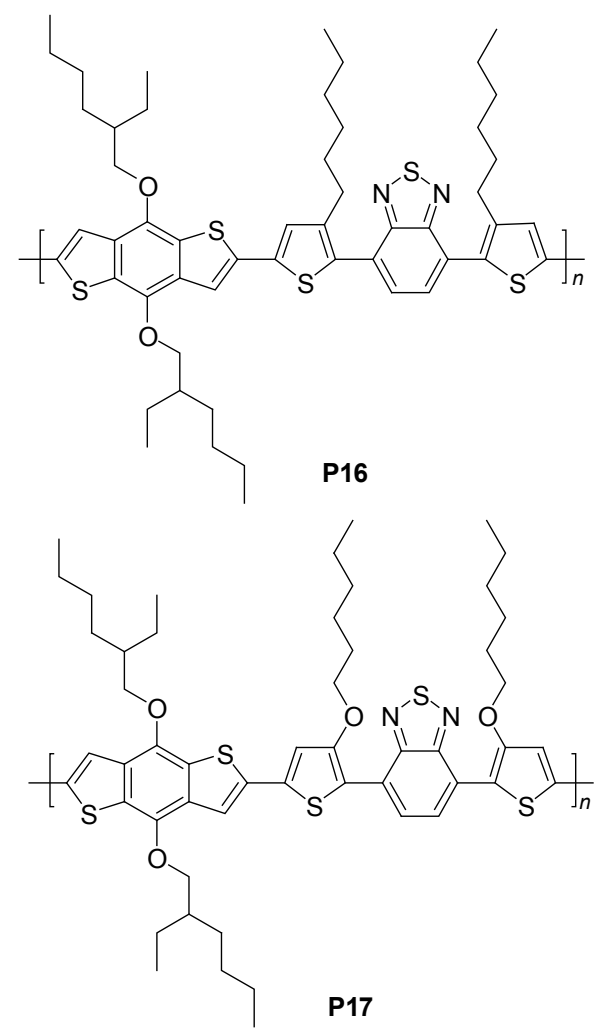

咔唑单元具有较强的给电子能力、优异的热稳定性 能及空穴传输性能 ${ }^{[59 \sim 62]}$, 此外, 氮原子上键接合适的烷 基链还可保证聚合物材料具有良好的溶解性. 喹喔啉类 (QX) 基团则具有较好的吸电子特性和共平面性 ${ }^{[63 \sim 65]}$, 因此将这些基团引入共轭聚合物主链中, 有望得到性能 优异的窄带隙聚合物. 为此, Andersson 等 ${ }^{[59]}$ 利用咔唑和 喹喔啉类 $(\mathrm{QX})$ 结构单元经 Suzuki 偶合制备了聚合物 $\mathbf{P 1 8}\left(M_{\mathrm{n}}=9000, \mathrm{PDI}=1.7\right)$, 并在 $\mathbf{P 1 8}$ 受体单元二苯喹喔 啉的结构基础上, 经辛氧基修饰制备出 P19 $\left(M_{\mathrm{n}}=\right.$ 23000, PDI = 2.7). 他们发现, 二苯喹喔啉上烷氧基的调 控对聚合物的能带隙和吸收光谱基本没有影响, 但却使
得聚合物的 $\mathrm{HOMO}$ 能级从 $-5.69 \mathrm{eV}$ 降至 $-5.73 \mathrm{eV}$, 开 路电压提高至 $0.92 \mathrm{~V}$, 但鉴于此特殊现象, 作者并未给 出合理的推论和解释. 在最优条件下制备的电池器件, P19 性能也优于 P18 (P18: $P C E=3.1 \% ; P 19: P C E=$ $3.7 \%)$.

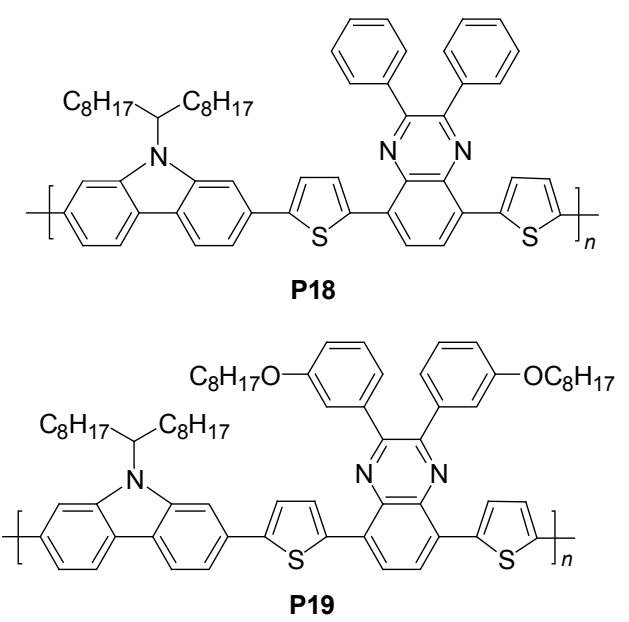

烷氧基调控并非对任何聚合物体系均有利, Chang 等 ${ }^{[66]}$ 做了深入的探究, 结合二环戊二烯苯并二硒吩 (IDS)与苯并噻二唑(BT)单元他们成功制备出共轭聚合 物 $\mathbf{P 2 0}\left(M_{\mathrm{n}}=10800, \mathrm{PDI}=2.0\right)$ 与 $\mathbf{P 2 1}\left(M_{\mathrm{n}}=10700, \mathrm{PDI}\right.$ $=1.7)$, 研究发现, $\mathbf{P 2 0}$ 与 $\mathbf{P 2 1}$ 的光学禁带宽度相同, 均 为 $1.6 \mathrm{eV}$, 紫外吸收光谱也无明显差异, 但 FET 测试显 示, 与 $\mathbf{P 2 0}$ 相比 $\left(\mathbf{P 2 0}: \mu_{\mathrm{h}}=8.0 \times 10^{-2} \mathrm{~cm}^{2} \cdot \mathrm{V}^{-1} \cdot \mathrm{s}^{-1}\right)$, 辛氧 基修饰的 P21 载流子迁移率只有 $2.6 \times 10^{-2} \mathrm{~cm}^{2} \cdot \mathrm{V}^{-1} \cdot \mathrm{s}^{-1}$. 相对较低的载流子迁移率使得激子复合几率增加, 电荷 在传输过程发生大量的能量损失, 进而不利于短路电流 $J_{\mathrm{sc}}$ 及器件能量转化效率 PCE 的提高, 导致同等条件下 制备电池器件, 器件性能也不如 P20 优异 $\left(\mathbf{P 2 1}: V_{\mathrm{oc}}=\right.$ $0.76 \mathrm{~V}, J_{\mathrm{sc}}=8.45 \mathrm{~mA} / \mathrm{cm}^{2}, \mathrm{FF}=41 \%, \mathrm{PCE}=2.6 \%$; P20: $V_{\mathrm{oc}}=0.76 \mathrm{~V}, J_{\mathrm{sc}}=11.24 \mathrm{~mA} / \mathrm{cm}^{2}, \mathrm{FF}=45 \%, \mathrm{PCE}=3.9 \%$ ). 基于 TD-B3LYP $/ 6-311 \mathrm{G}(\mathrm{d}, \mathrm{p})$ 模型, 通过理论计算他们 发现, 在侧链方向上, 聚合物 P20 体系中, 由己基与苯 环组成的苯己烷侧链形成了二面角为 $23.1^{\circ}$ 的面外构象, 而 P21 中烷氧基与苯环则趋近于共平面，二面角仅有一 $1.9^{\circ}$, 无疑从热力学角度可以判定 $\mathbf{P} 21$ 处于较高能态, 与此同时, P21 中 $\mathrm{O}$ 原子与苯环的 $\mathrm{p}-\boldsymbol{\pi}$ 共轭, 又易诱导侧 链重叠构象的产生，从而影响高分子链的聚集，抑制电 荷的有效传输, 降低其载流子迁移率.

与长链烷基相似, 烷氧基侧链位置的选择与调控亦

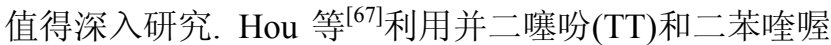
啉(DBQX)单元, 通过对受体单元烷氧基侧链位置进行 调控, 制备出聚合物 P22 与 $\mathbf{P 2 3}$. 测试结果显示, P22 与 P23 的吸收光谱基本没有差异, 光学与电化学禁带宽度 


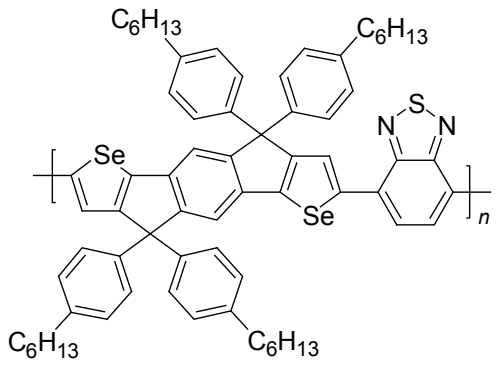

P20

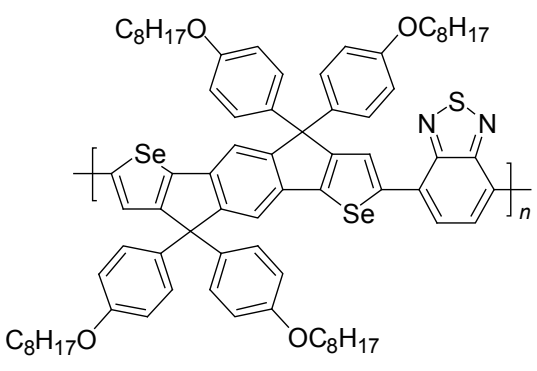

P21

亦相差不大. 采用 B3LYP/6-31G(d,p)方法, 发现对位的 烷氧基具有更强的给电子效应，从而使得 P23 获得更高 的 HOMO 与 LUMO 轨道能级(分别为 -5.01 和 -3.54 $\mathrm{eV}$ ). 由于开路电压 $V_{\mathrm{oc}}$ 主要取决于给体材料的 HOMO 能级与受体材料 LUMO 能级之间的能级差, 所以给体 材料较高的 HOMO 能级无疑限制了光电转化效率的提 升, 最终导致基于 $\mathbf{P 2 2}: \mathrm{PC} 71 \mathrm{BM}=1: 3$ 比例混合制备 的太阳能电池器件显示出更低的光电性能, 电流密度和 开路电压难以进一步提高 $\left(\mathbf{P 2 2}: V_{\mathrm{oc}}=0.60 \mathrm{~V}, J_{\mathrm{sc}}=8.22\right.$ $\mathrm{mA} / \mathrm{cm}^{2}, \mathrm{FF}=0.594, \mathrm{PCE}=2.93 \% ; \mathbf{P 2 3}: V_{\text {oc }}=0.73 \mathrm{~V}$, $\left.J_{\mathrm{sc}}=9.87 \mathrm{~mA} / \mathrm{cm}^{2}, \mathrm{FF}=0.551, \mathrm{PCE}=3.97 \%\right)$.

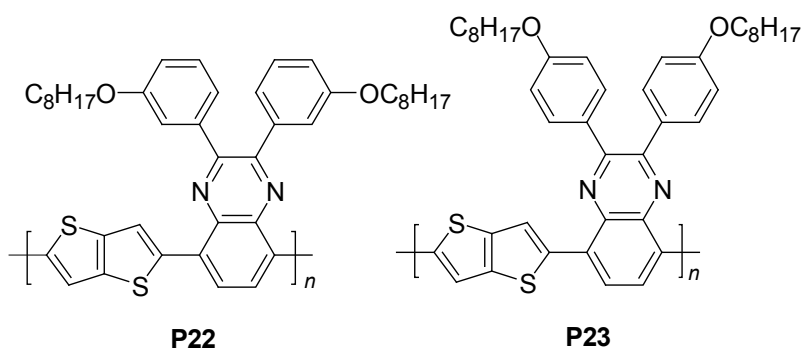

\subsection{2 拉电子型侧链}

根据前线轨道理论, 吸电子基团的引入可有效降低 聚合物 HOMO 与 LUMO 的轨道能量 ${ }^{[24]}$. 较低的 HOMO 有利于提高器件开路电压 $V_{\mathrm{oc}}$, 然而低的 LUMO 能级则 可能会导致电子传输的驱动力不足, 从而会增加电荷复 合概率, 不利于载流子传输 ${ }^{[33 ~ 35,68]}$. 因此, 合理的分子 设计既要综合考虑器件的开路电压 $V_{\mathrm{oc}}$, 又要平衡电子 传输效率, 避免电荷复合损失. 而目前, 广泛应用于共 轭聚合物体系中拉电子型侧链主要有酯基和羰基等.
聚噻吩乙烯(PTV)衍生物作为一种窄能带隙 $(1.5$ $1.8 \mathrm{eV}$ )共轭聚合物材料, 具有良好的导电性和优异的空 穴传输性能 $\left(0.22 \mathrm{~cm}^{2} \cdot \mathrm{V}^{-1} \cdot \mathrm{s}^{-1}\right)$ 等特点, 在有机场效应晶 体管、有机太阳能电池及非线性光学材料等方面亦具有 广泛的应用 ${ }^{[69 \sim 72]}$. 可是, 其光伏特性表现一般，已报道 的 P24 与 PCBM共混制备的光伏器件效率只有 $0.02 \%$ 左 右 ${ }^{[73]}$. 为改善 PTV 光电性能, Huo 等 ${ }^{[74]}$ 在芳环上化学修 饰引入吸电子基团, 制备出酯基取代的 P25 $\left(M_{\mathrm{w}}=\right.$ $16500, \mathrm{PDI}=2.8)$, 研究发现, $\mathbf{P 2 4}$ 与 $\mathbf{P 2 5}$ 在 $\mathrm{CHCl}_{3}$ 溶液 和薄膜状态下具有相似的光谱性能, 其次, 诸如 P24 类的 PTV 衍生物在溶液状态下一般无荧光性质，但 P25 却具有较强的苂光，作者推测此现象无疑归因于酯基的 化学修饰作用, 具体表现在: (1)减少了激发态电子非辐 射跃迁去活化返回基态这一过程的途径; (2)增加了激发 态电子的寿命, 利于诱导电荷转移至 PCBM 受体处, 降 低了载流子复合几率; (3)酯基的吸电子效应，可增强分 子内电荷转移, 促进激子分离.

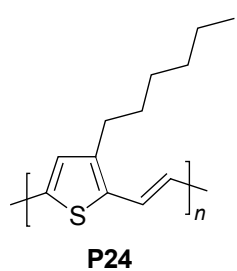

P24

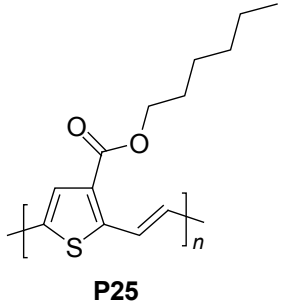

P25
与此同时, 酯氧羰基的吸电子作用, 还引起了共轭 聚合物分子自身电子结构的变化，相较 P24，P25 的 $\mathrm{HOMO}$ 与 $\mathrm{LUMO}$ 能级均降低了 $0.21 \mathrm{eV}$, 使得器件的开 路电压从 $0.54 \mathrm{~V}$ 提高至 $0.82 \mathrm{~V}$, 更高的空穴迁移率 $(\mathbf{P 2 4}$ : $\mu_{\mathrm{h}}=1.6 \times 10^{-3} \mathrm{~cm}^{2} \cdot \mathrm{V}^{-1} \cdot \mathrm{s}^{-1} ;$ P25: $\mu_{\mathrm{h}}=3.1 \times 10^{-3} \mathrm{~cm}^{2}$ • $\mathrm{V}^{-1} \cdot \mathrm{s}^{-1}$ )也使得器件的短路电流增至 $4.78 \mathrm{~mA} / \mathrm{cm}^{2}$, 本体 异质结太阳能电池器件性能也得到了大幅改善, 光电转 化效率从 $0.02 \%$ 提高至 $2.01 \%$.

Liang 等 ${ }^{[75]}$ 将苯并二噻吩与酯基取代的并二噻吩单 元共聚, 得到的聚合物具有较高 HOMO 能级, 较好的空 穴迁移率和自组装性能, 使得基于此聚合物与 PC71BM 共混制备的电池光电转换效率达到 5.6\%. 随后，Hou 等 ${ }^{[76]}$ 为调控并二噻吩基团的吸电子能力, 通过对受体 单元进行侧链修饰，将酯基换成酮基，发现其 HOMO 能 级从 $-5.01 \mathrm{eV}$ 下移至 $-5.12 \mathrm{eV}$, 而后，又通过氟原子 取代 P27 导致 HOMO 能级进一步下移至 $-5.22 \mathrm{eV}$. 光 伏器件的测试结果充分证明了下调给体材料 HOMO 能 级的策略对提高聚合物太阳能电池开路电压的有效性. 开路电压从 P26 的 $0.67 \mathrm{~V}$ 提高至 P28 的 $0.76 \mathrm{~V}$, 能量转 换效率也从 $\mathbf{P 2 6}$ 的 5.15\%提高到 $\mathbf{P 2 8}$ 的 7.7\%.

综上, 吸电子型侧链的引入可有效降低聚合物材料 

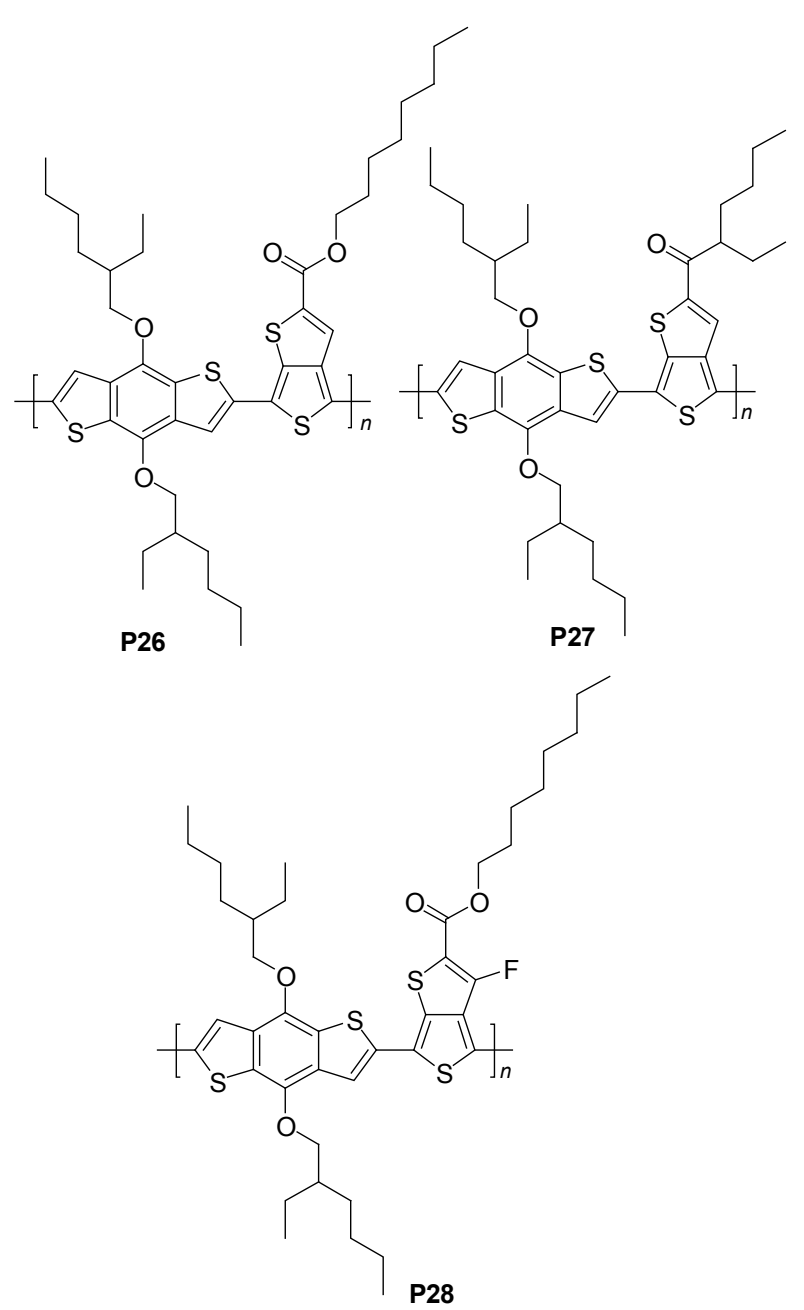

HOMO 与 LUMO 的轨道能量, 从而改善器件的开路电 压 $V_{\mathrm{oc}}$.

\section{2 .3 共轭型侧链}

为降低聚合物材料带陌, 拓宽光谱响应, 提高材料 光电性能, 近年来, 大量新型的给受体型共轭聚合物材

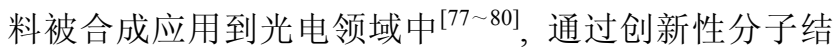
构设计, 一方面可增加主链共轭度, 使吸收光谱红移、 能带隙减小, 光学吸收性能增强 ${ }^{[11 ~ 83]}$; 其次, 可在统筹 主链结构平面性与侧链位阻情况下, 通过延长支链共 轭, 拓宽光谱响应范围, 提高太阳光的利用率 ${ }^{[84,92]}$.

苯并二噻吩(BDT)单元具有较大的刚性共轭平面, 有利于聚合物分子主链的 $\pi-\pi$ 堆积 ${ }^{[86,87]}$, 基于 BDT 聚合 物表现出的优异性能, 目前此类聚合物常用于有机光电 材料. Hou 等 ${ }^{[88}$ 从侧链调控出发, 以辛基噻吩取代辛氧 基为链接基团对苯并二噻吩(BDT)单元进行结构优化, 继而制备线型共轭聚合物 $\mathbf{P 3 0}\left(M_{\mathrm{n}}=17000, \mathrm{PDI}=3.6\right)$, 研究发现侧链共轭强度的增强并未对吸收光谱产生预 设的影响, 但聚合物的 HOMO 轨道能级却发生了一定 的变化, 由 $-4.98 \mathrm{eV}$ 降至 $-5.21 \mathrm{eV}$, 降低了 $0.23 \mathrm{eV}$, 采用 DFT(B3LYP/6-31G)方法, 对聚合物分子的几何构
型和电子结构分析发现: (1) P30 的 HOMO 轨道可离域 至辛基噻吩侧链中，电子离域性显著增强; (2)较 $\mathrm{S}$ 原子, $\mathrm{O}$ 原子直径较小, 故在苯并二噻吩 (BDT) 与苯并二呋喃 (BDF)体系中, 较小的体积效应, 使得 BDF 中侧链与骨 架的位阻效应减弱, 扭转角较小, 空间位阻小的构建单 元能使相应聚合物分子间排列更为紧密. 与 PC71BM以 $1: 1.5$ 比例的本体异质结电池器件性能测试结果显示, P29 与 P30 短路电流与填充因子相差很小, 但基于较低 的 HOMO 能级, 形成较大的开路电压, 使得 P30 器件性 能更为优越, 效率达 $6.26 \%\left(\mathbf{P 2 9}: V_{\mathrm{oc}}=0.63 \mathrm{~V}, J_{\mathrm{sc}}=\right.$ $13.87 \mathrm{~mA} / \mathrm{cm}^{2}, \mathrm{FF}=0.597, \mathrm{PCE}=5.22 \% ; \mathbf{P 3 0}: V_{\mathrm{oc}}=0.78$ $\mathrm{V}, J_{\mathrm{sc}}=13.04 \mathrm{~mA} / \mathrm{cm}^{2}, \mathrm{FF}=0.61, \mathrm{PCE}=6.26 \%$ ).
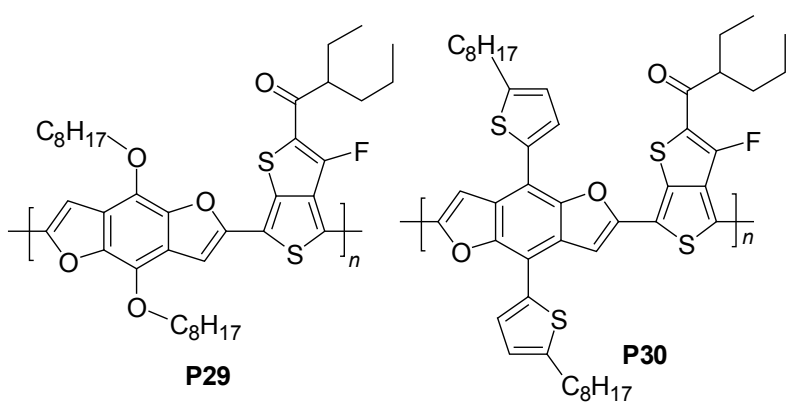

随后，他们 ${ }^{[89]}$ 又在上述基础上成功制备了线型聚 合物 P31 与 P32, 比较发现, P32 的吸收光谱约红移 20 $\mathrm{nm}$, 能带隙、HOMO 和 LUMO 轨道能级与 $\mathbf{P 3 1}$ 几乎一 致, SCLC 测试表明, P31 与 P32 的空穴迁移率相差亦不 大, 分别为 $4.48 \times 10^{-2}$ 和 $2.35 \times 10^{-2} \mathrm{~cm}^{2} \cdot \mathrm{V}^{-1} \cdot \mathrm{s}^{-1}$. 但是, 与 $\mathbf{P 3 1}$ 相比, 基于 $\mathbf{P 3 2}$ 自身较高的表面能以及与受体材 料较好的相容性, 使得聚合物 P32 与 PC71BM 混合膜形 貌的相分离尺寸较小, 便于纳米级互渗双连续网络结构 的形成, 电池器件优化条件下效率可达 $4.23 \%$. 同年, Kwon 等 $^{[00]}$ 也研究了给体单元为二噻吩连苯并二噻吩 (DTBDT)时侧链调控的影响，他们发现，侧链结构优化 后, 聚合物材料 P34 的吸收光谱在溶液和薄膜状态下均 蓝移 $10 \mathrm{~nm}$ 左右, 但欣喜的是, 良好的分子链聚集性使 得薄膜状态下的 P34 在长波段有明显的肩峰. SCLC 测 试显示, P34 载流子迁移率竟高达 $0.55 \mathrm{~cm}^{2} \cdot \mathrm{V}^{-1} \cdot \mathrm{s}^{-1}$ $\left(\right.$ P33: $0.001 \mathrm{~cm}^{2} \cdot \mathrm{V}^{-1} \cdot \mathrm{s}^{-1}$ ), 作者推测较高的载流子迁移 率可能归因于相邻共轭链间轨道重叠区域的增加, 基于 优良的空穴迁移性能, P34 与 PC71BM 混合制成的光电 器件, 优化后效率达 $5.1 \%$.

随着研究的深入, Chen 等 ${ }^{[91]}$ 又着眼于聚合物 $\pi$ 桥的 侧链调控, 在 P35 的结构基础上, 在噻吩链接基团键接 己基噻吩制备出 P36. 发现共轭的噻吩侧链对光学禁带 宽度影响不大, 但对聚合物的 HOMO 能级影响较大, 导 致 P36 的 HOMO 减小 $0.27 \mathrm{eV}$. 作者推测, 由于侧链的 


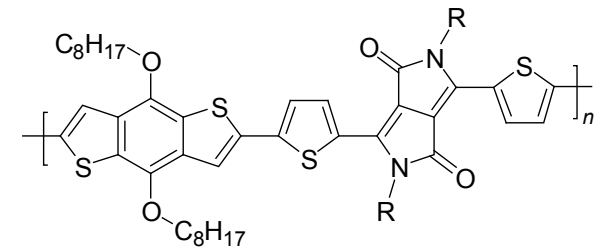<smiles>[R]CC(CC)CCCCCCCCC</smiles>

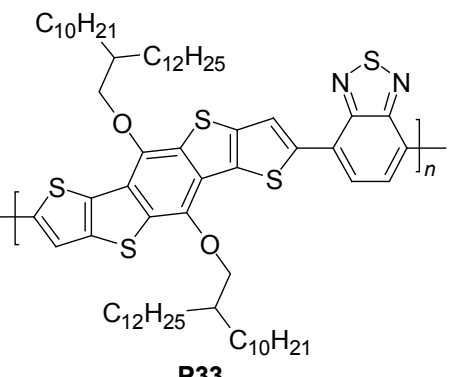

P33

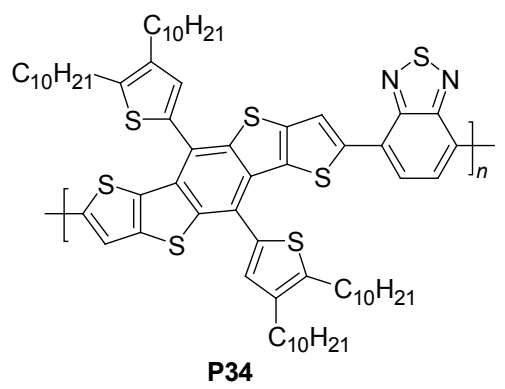

不利位阻, 以及噻吩侧链与 IDT 单元中硫原子之间较强 的静电作用, 使得主链扭曲度增大, 导致主链共轭度降 低. 基于更低的 HOMO 轨道, P36 获得了较为优异的性 能, 和 P71BM 配合制备的本体异质结电池, 优化后, $V_{\mathrm{oc}}=0.93 \mathrm{~V}, J_{\mathrm{sc}}=9.1 \mathrm{~mA} / \mathrm{cm}^{2}, \mathrm{FF}=52.7 \%$, 器件效率达 $4.5 \%$.

聚噻吩类衍生物也是被广泛研究的有机光电材 料 ${ }^{[92]}$. 其中规整的 P3HT, 由于良好的溶解性、成膜性 能、结晶性外加较高的载流子迁移率, 使得基于 P3HT 与 PC61BM 的太阳能电池器件效率已超过了 $5 \%{ }^{[93,94]}$, 但 P3HT 自身较弱的光吸收能力外加较高的 HOMO 能 级阻碍了效率的进一步提高 ${ }^{[95,96]}$. 为增加 P3HT 侧链的 共轭长度, 拓宽光谱响应范围, 李永舫等 ${ }^{[97]}$ 在此领域做 了大量而深入的研究工作, 他们通过在聚合物主链上引<smiles>[R]C1([R])c2cc3c(cc2-c2sc(-c4ccc(-c5ccc(C(C)(C)C)c6nsnc56)s4)cc21)C([R])([R])c1cc(-c2ccc(C(C)(C)C)s2)sc1-3</smiles>

P35

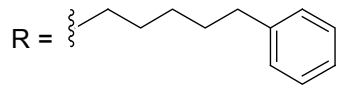

$\mathrm{C}_{6} \mathrm{H}_{13}$

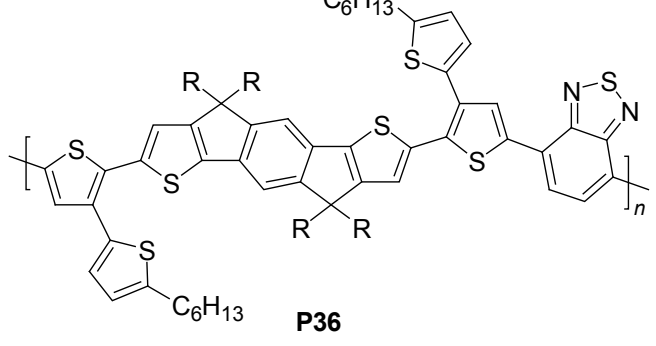

入共轭支链的方式, 制备出聚合物 $\mathbf{P 3} 7^{[98]}$, 但由于共轭 侧链的较大位阻, 主链的扭曲导致了较差的光吸收性 能. 为拓展支链的共轭长度, 把苯乙烯延长为二联(苯 乙烯)使得 P38 吸收带红移至可见光区，为降低含共轭 支链的噻吩单元浓度以调控其对主链扭曲的影响制备 的 P39 使得聚合物在 380 至 $650 \mathrm{~nm}$ 范围内呈现出良好 的光谱响应.
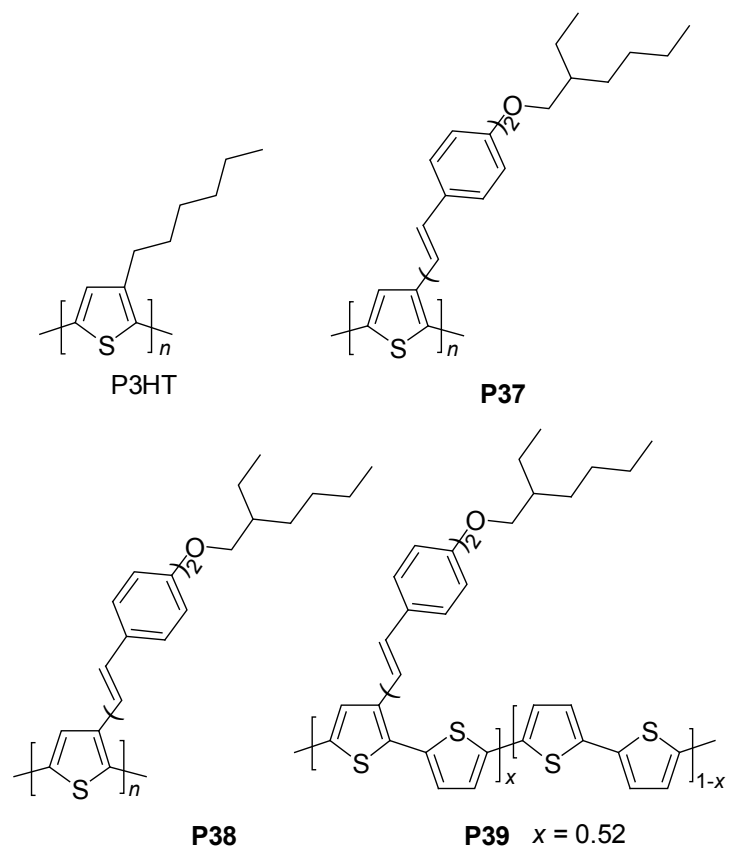

为进一步改善材料光吸收性能，使聚合物在可见光 区域达到全吸收，随后，他们又在统筹位阻效应、主链 结构平面性及支链共轭度的基础上，对聚合物分子结构 进行优化设计，把共轭支链的苯乙烯换成噻吩乙烯制备 出了窄能带隙聚噻吩衍生物 P40, 研究发现 P40 薄膜状 态下具有较宽的光谱响应，在 $350 \mathrm{~nm}$ 到 $650 \mathrm{~nm}$ 范围内 显示一个宽的吸收平台，并且在热淬火后，也表现出良 
好的自组装性能, 吸收峰更是拓宽至 $700 \mathrm{~nm}$, SCLC 结 果显示其空穴迁移率达 $3.7 \times 10^{-4} \mathrm{~cm}^{2} \cdot \mathrm{V}^{-1} \cdot \mathrm{s}^{-1}$.

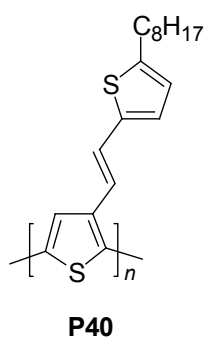

随后制备的聚合物 $\mathbf{P 4 1} \sim \mathbf{P 4 3}^{[99]}$, 其光物理性能与 P3HT 相比也得到了改善, 薄膜的吸收光谱在 P3HT 吸 收很弱的 $350 \sim 450 \mathrm{~nm}$ 波长范围都出现了较强的吸收 峰. 尤其是聚合物 P43, 在 $350 \sim 660 \mathrm{~nm}$ 波长范围更是 呈现出一个近似的全吸收. 除此之外, 聚合物 P43 的电 子能级也发生了变化, HOMO 能级较 P3HT 下降了 0.17 $\mathrm{eV}$, 有益于光伏器件开路电压的提高. 基于 $\mathbf{P 4 3}$ 与 PCBM 共混制备的聚合物太阳能电池, 在 AM1.5, 100 $\mathrm{mW} / \mathrm{cm}^{2}$ 光照条件下器件开路电压为 $0.72 \mathrm{~V}, \mathrm{PCE}$ 达到 $3.18 \%$. 而相同条件下, 基于 $\mathrm{P} 3 \mathrm{HT} / \mathrm{PCBM}$ 的光伏器件能 量转换效率却只有 $2.41 \%$, 开路电压仅有 $0.6 \mathrm{~V}$.

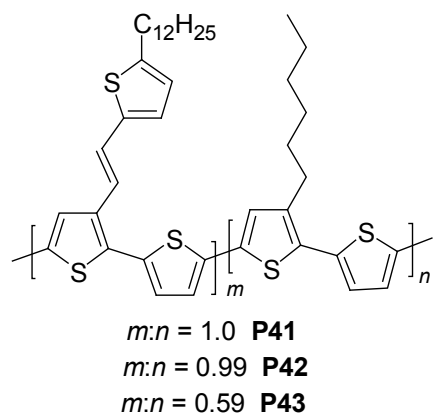

为了进一步研究给体一受体(D-A)型聚合物材料结 构与性能之间的关系, 改善聚合物材料的光电性能, Huang 等 ${ }^{[100]}$ 创造性的提出了一种新的分子结构设计策 略, 以给体单元与 $\pi$ 桥为聚合物主链骨架, 将受体单元 以共轭方式键接于侧链, 制备出新型的侧链 D-A 型聚合 物. 与主链 D-A 型聚合物相比, 侧链 D-A 型聚合物材料 具备如下优点: 首先, 侧链 D-A 型结构不仅可利用分子 内电荷转移调节聚合物的吸收光谱, 而且还可促进聚合 物主链上各向同性的电荷传输, 促使电子在侧链富集, 有利于电荷分离; 其次, 通过对侧链分子结构的调控设 计, 可得到较低的 HOMO 能级, 提高器件的开路电压; 再者, 二维的分子链结构还可有效改善聚合物材料的溶 解性能，提高材料的成膜与自组装性能.

近几年来，一系列此类型聚合物材料被设计合成出 来, 最高的能量转化效率已经超越了 $6 \%{ }^{[101 ~ 103]}$.
谭松庭等 ${ }^{[104]}$ 在此领域做了大量的基础性研究工作, 他们以苯并二噻吩与噻吩桥为共轭主链, 对侧链单元进 行系统的分子结构设计, 制备出具有不同共轭侧基的噻 吩一苯并二噻吩的侧链 D-A 型共聚物 P44 $\left(M_{\mathrm{n}}=121000\right.$, $\mathrm{PDI}=2.2), \mathbf{P 4 5}\left(M_{\mathrm{n}}=202000, \mathrm{PDI}=2.6\right)$ 与 $\mathbf{P 4 6}\left(M_{\mathrm{n}}=\right.$ $125000, \mathrm{PDI}=1.8)$. 电化学测试显示, P44 P46 的 $\mathrm{HOMO}$ 轨道能级分别为 $-5.22,-5.44$ 和 $-5.25 \mathrm{eV}$, 随 着侧链共轭长度的增加, 主链骨架上的电子也更趋于离 域, 致使 LUMO 能级分别降至- $3.40,-3.44$ 以及一 $3.54 \mathrm{eV}$. 结合器件的电流密度-电压 $(J-V)$ 、波长-外量子 效率特性曲线以及器件性能的差异反映出不同侧链对 聚合物材料的光电性能有很大影响. OFET 测试表明, P45 的空穴迁移率达 $3.2 \times 10^{-3} \mathrm{~cm}^{2} \cdot \mathrm{V}^{-1} \cdot \mathrm{s}^{-1}$, 比 P44 与 P46 高出近两个数量级, 可见光区域较好的光谱响应、 较低的 $\mathrm{HOMO}$ 能级以及优异的载流子迁移性能, 使得 基于 ITO/PEDOT:PSS/P45:PC61BM $(1: 4, w / w) / \mathrm{Ca} / \mathrm{Al}$ 器件结构的 $J_{\mathrm{sc}}=12.65 \mathrm{~mA} / \mathrm{cm}^{2}, V_{\mathrm{oc}}=0.81 \mathrm{~V}, \mathrm{FF}=0.41$, PCE 达 $4.18 \%$.

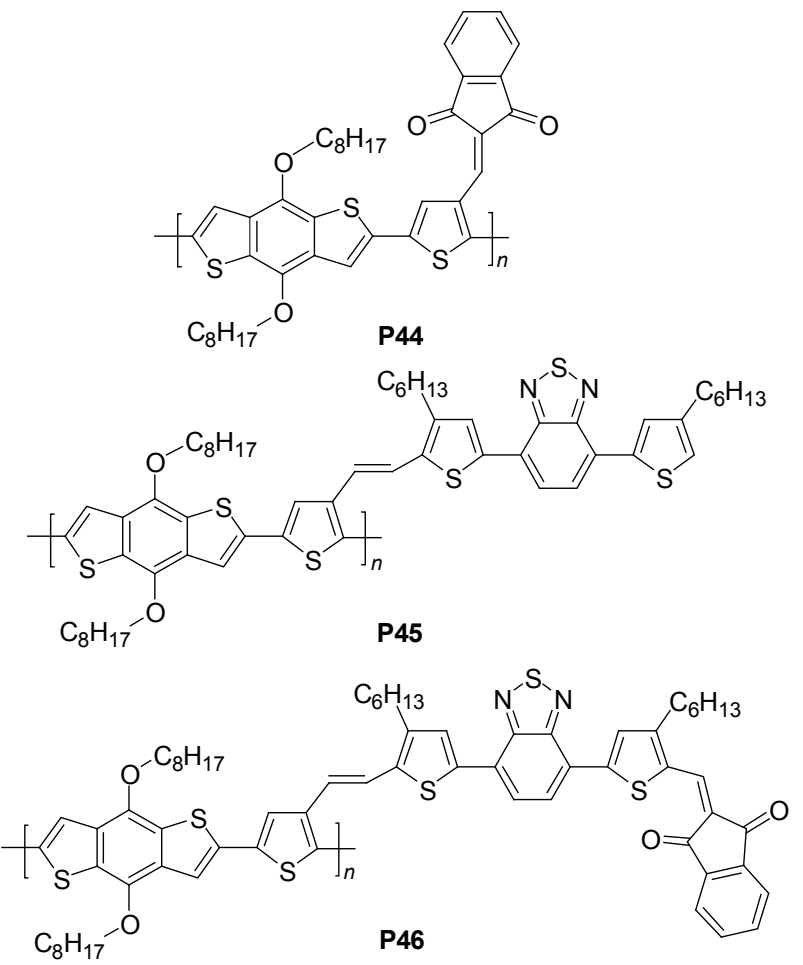

基于分子侧链较强的可调控性特点与优势, 次年, 他们 ${ }^{[105]}$ 又在 P47 $\left(M_{\mathrm{w}}=157000, \mathrm{PDI}=1.81\right)$ 的结构基础 上成功制备出 $\mathbf{P 4 8}\left(M_{\mathrm{w}}=124000, \mathrm{PDI}=1.26\right)$ 与 $\mathbf{P 4 9}$ $\left(M_{\mathrm{w}}=514000, \mathrm{PDI}=2.33\right) . \mathrm{B} 3 \mathrm{LYP} / 6-31 \mathrm{G}(\mathrm{d}, \mathrm{p})$ 理论计算 结果显示, 与 $\mathbf{P 4 8}$ 相比, P49 主链结构中的苯并二噻吩 $(B D T)$ 与噻吩单元构成的二面角仅为 $2.8^{\circ}$, 此外, 主侧 链噻吩间的二面角也减小至 $1^{\circ}$, 无疑暗示着 $\mathbf{P 4 9}$ 给受体 单元间具有更为优良的分子共平面性和更强的分子间 
相互作用. 此推论在光物理性能测试中也找到了佐证, 在归因于分子内电荷转移(ICT) 的低能长波段, 与 $\mathbf{P 4 8}$ 相比, P49 在溶液和薄膜状态下的最大吸收波长分别红 移了 37 和 $27 \mathrm{~nm}$, 薄膜状态下的吸收边更是达到了 776 $\mathrm{nm}$. 而由于 P49 中 BDT 单元侧链噻吩的较大位阻, 使 得与烷氧基侧链的 P48 相比, 共轭效应反而减弱, 故而 P49 在归结于主链离域的 $\pi-\pi$ *电子转变的高能低波数段 发生了小幅度的蓝移. SCLC 测试结果显示 P49 的空穴 迁移率最为出色, 达到 $1.2 \times 10^{-2} \mathrm{~cm}^{2} \cdot \mathrm{V}^{-1} \cdot \mathrm{s}^{-1}$, 因此, 基 于 ITO/PEDOT:PSS/P47-49:PC61BM/LiF/Al 的器件结 构, P49 获得了更高的转换效率, $V_{\mathrm{oc}}=0.64 \mathrm{~V}, J_{\mathrm{sc}}=6.21$ $\mathrm{mA} / \mathrm{cm}^{2}, \mathrm{FF}=0.42, \mathrm{PCE}=1.68 \%\left(\mathbf{P} 47: V_{\mathrm{oc}}=0.50 \mathrm{~V}\right.$, $J_{\mathrm{sc}}=8.16 \mathrm{~mA} / \mathrm{cm}^{2}, \mathrm{FF}=0.33, \mathrm{PCE}=1.34 \% ; \mathbf{P 4 8}: V_{\mathrm{oc}}=$ $\left.0.52 \mathrm{~V}, J_{\mathrm{sc}}=3.51 \mathrm{~mA} / \mathrm{cm}^{2}, \mathrm{FF}=0.31, \mathrm{PCE}=0.56 \%\right)$.

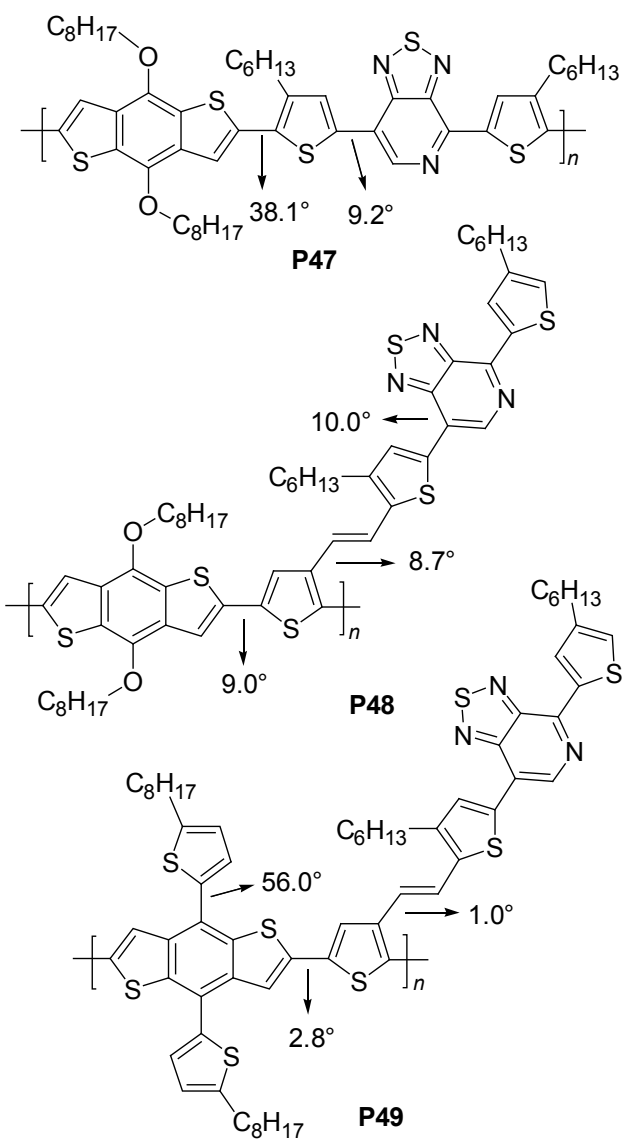

曹锯研究组 ${ }^{[106]}$ 则利用强吸电子能力的双噻吩二酮 吡咯并吡咯(DPP)结构单元, 在 P50 的结构基础上, 制 备出新型的侧链 D-A 型聚合物 P51 $\left(M_{\mathrm{n}}=134000\right.$, PDI = 1.27). 出色的溶解性能、较低的 HOMO 能级 $(-5.34 \mathrm{eV})$ 加上优异的载流子迁移能力, 使得与 PC71BM 混合制成的本体异质结器件, 光电转换效率高 达 4.89\%. 随后, Tan 等 ${ }^{[107]}$ 又利用更为新颖的异靛蓝结 构单元成功制备出 P52, 优化器件结构后其 PCE 更是高 达 $6.51 \%$, 这也是到目前为止, 基于侧链 D-A 型结构的
聚合物材料所获得的最高光电转换效率.

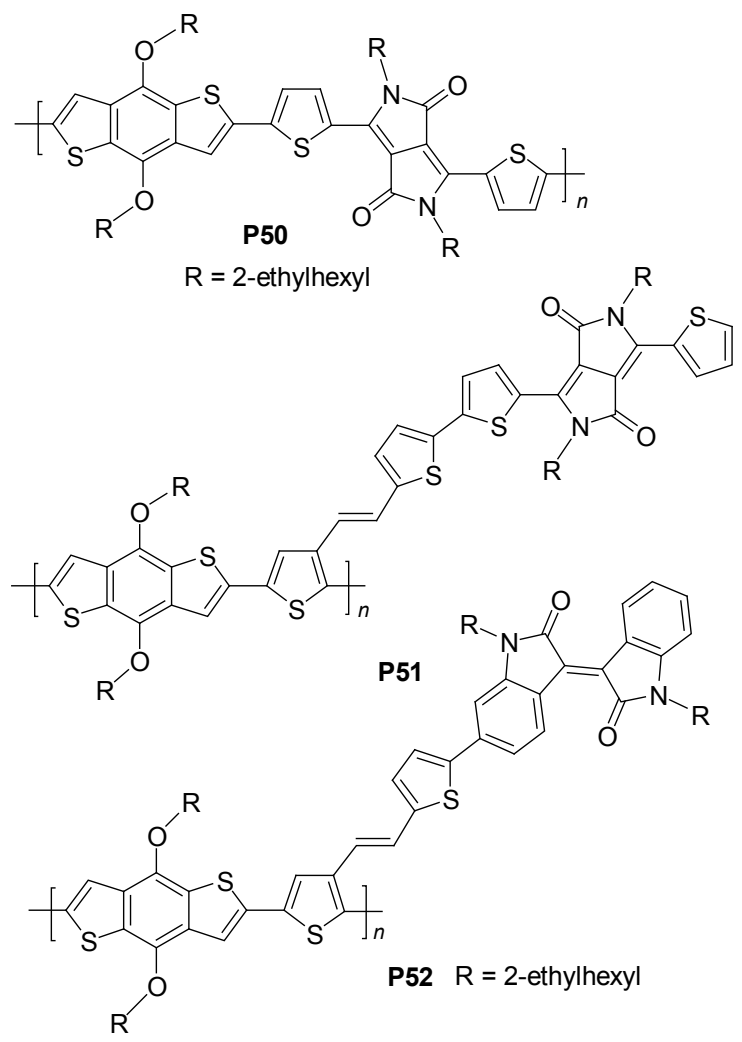

然而, 与主链 D-A 型聚合物材料相比, 基于侧链 D-A 型聚合物器件的光电转换效率依然较低, 其中的深 层次原因可能是与混合膜形貌尚待调控有关 ${ }^{[108]}$. 因此, 亟需制备出新型的侧链 D-A 型聚合物材料, 以系统研究 分子结构与混合膜形貌、光电性能之间内在规律.

\section{3 水/醇溶性侧链}

与此同时, 聚合物光电器件中各功能层间的界面形 态亦是影响器件性能的关键因素 ${ }^{[109]}$, 在处理环节过程 中, 最为关键的就是对活性层/电极界面的优化. 电极缓 冲层(修饰层)的加入，可大幅降低活性层与电极的接触 势垒, 有效提升载流子收集率. 近年来, 有关侧链水/醇 溶性的共轭聚合物在光电器件中研究与应用亦引起了 显著关注 ${ }^{[110,111]}$. 侧链水/醇溶共轭聚合物与传统侧链共 轭聚合物相比，因其独特的物理性质和优异的界面性能 已作为界面修饰或活性材料广泛应用在光电器件中 ${ }^{[112]}$. 研究发现，因侧链水/醇溶共轭聚合物中的极性侧链基 团的作用，使得此类材料在水或醇等极性溶剂中均有良 好的溶解性能, 故而可有效地克服器件加工过程中的界 面互溶现象 ${ }^{[113]}$. 除此之外, 在多层结构的光电器件中, 还可以有效的改善电极与有机活性层间的电子传输或 收集, 从而大幅的提高器件光电性能 ${ }^{[14]}$. 下文中我们 将综述系列新型侧链水/醇溶共轭聚合物材料在光电器 件中的应用. 研究表明, 基于此类新材料的聚合物电致 
发光器件以及聚合物太阳电池的性能均可得到大幅提 高 ${ }^{[115,116]}$.

水/醇溶性侧链修饰的共轭聚合物材料因其可有效 防止蒸镀的阴极金属对活性层的污染; 优化活性层与金 属电极的接触界面, 减小器件的并、串联电阻; 有效提 高器件的内建电势等优点, 且自身在活性层与金属电极 界面处的特殊排列还可形成特定取向的偶极子, 有利于 减小载流子注入的势垒 ${ }^{[117]}$. 因此, 近年来, 国内外学者 在此领域做了大量的研究与尝试 ${ }^{[118,119]}$.

\subsection{1 非离子型水/醇溶性侧链}

鉴于极性溶剂中优异的溶解性能以及与金属离子 良好的配位性能, 多乙二醇型侧链亦常应用于有机聚合 物光电材料中. 但是在极性溶剂中高度的分散性使得多 乙二醇型侧链修饰的聚合物可与水发生较强的相互作 用, 从而影响 OPV 与 OFET 器件的稳定性及光电效

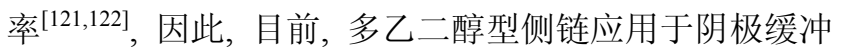
层材料中的报道较少. Xiao 等 ${ }^{[120]}$ 报道了多乙二醇型侧 链修饰的 P53, 研究发现, 与 P3HT 相比, P53 的空穴迁 移率只有 $3.5 \times 10^{-5} \mathrm{~cm}^{2} \cdot \mathrm{V}^{-1} \cdot \mathrm{s}^{-1}$, 低于室温的玻璃化转 变温度也导致了薄膜形貌呈现较差的稳定性.

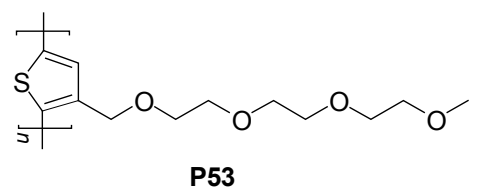

而 Patil 等 ${ }^{[121]}$ 发现烷基链与多乙二醇型链的协同修 饰作用可获得较为理想的效果, 基于 P54 的 OFET 测试 显示电流开关比达 $10^{4}$ 数量级, 电子迁移率更是达到了 $3 \mathrm{~cm}^{2} \cdot \mathrm{V}^{-1} \cdot \mathrm{s}^{-1}$. 次年, Hayward 与 Emrick 等 ${ }^{[122]}$ 利用非极 性己基与极性三乙二醇(TEG)键接在相邻噻吩侧链处成 功制备出双嵌段共聚物 P55, 研究发现, 由于相邻嵌段 侧链间的己基与三乙二醇相互作用，使得聚合物在甲醇 溶液中可有效自组装成有序的纳米纤维状结构, 此外, $\mathrm{K}^{+}$与 TEG 的配位作用还可诱导聚合物材料呈现超螺旋 结构. 这一发现, 为聚合物材料的自组装性能、构建良 好的共混膜形貌以及光电器件性能改善研究提供了一 个新的思路.

基于 ITO/P56/PCDTBT12:PC71BM/ $/ \mathrm{MoO}_{3} / \mathrm{Al}$ 反转 型器件结构, $\mathrm{CaO}$ 等 ${ }^{[123]}$ 利用侧链含有磷酸酯的均聚物 P56 作为阴极修饰层, 光物理、电化学及器件性能测试 显示, 短路电流达 $10.68 \mathrm{~mA} / \mathrm{cm}^{2}$, 器件 PCE 更是高达 $6.04 \%$. 次年, Chen 等 ${ }^{[124]}$ 成功制备了侧链为非离子亲水 型的聚噻吩衍生物 P57, 基于 ITO/ZnO/P57/P3HT: PCBM/PEDOT:PSS/Ag 反转型器件结构, 器件测试结果 也显示, 与单一的 $\mathrm{ZnO}$ 相比, $\mathrm{ZnO} / \mathbf{P 5 7}$ 的器件转换效率

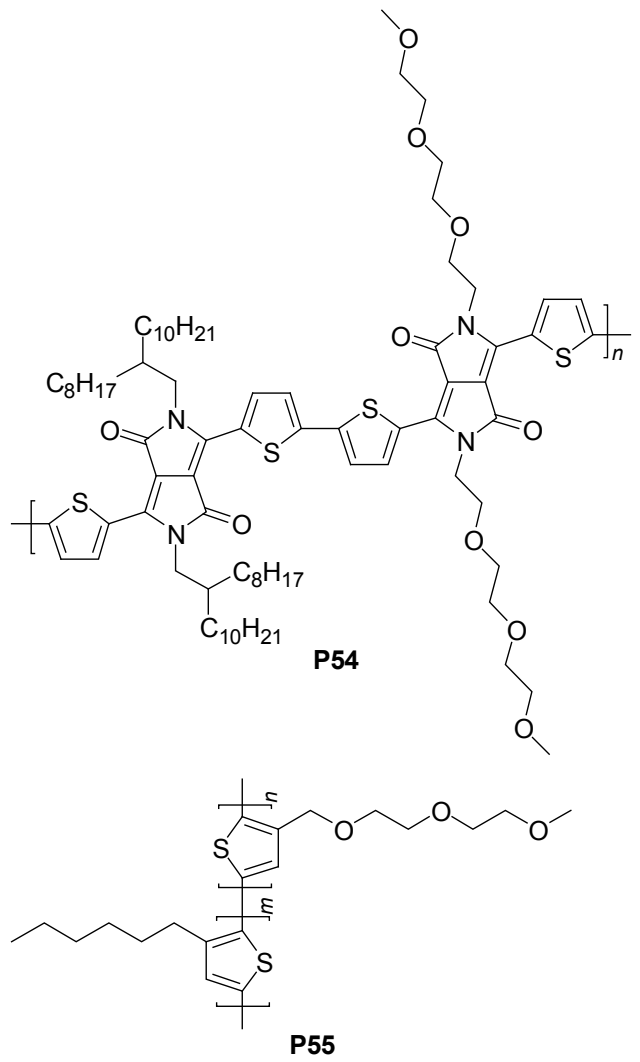

从 $2.99 \%$ 提高至 $3.98 \%$.
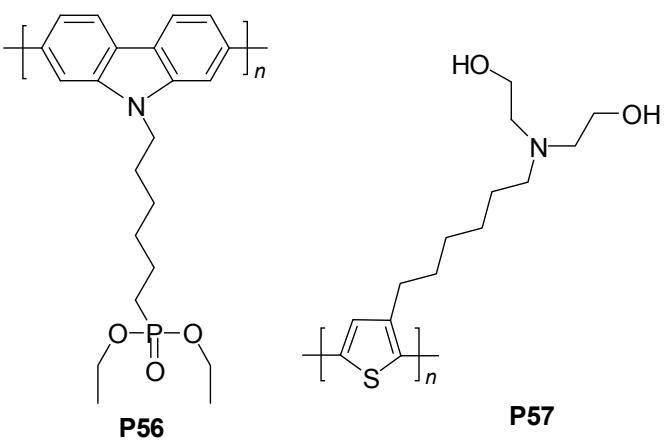

1.3.2 离子型水/醇溶性侧链

2009 年 $\mathrm{Kim}$ 等 ${ }^{[125]}$ 在聚芴(PPV)侧链末端引入离子 型氨基, 作为阴极缓冲层对光电材料活性层与金属电极 接触界面进行了优化, 在传统本体异质结器件 ITO/ EDOT/P3HT:PCBM/P58/Al 结构基础上, 发现在活性层 和阴极铝之间旋涂一层 3 5 nm 的 P58 修饰层后, 器件 与单一 $\mathrm{Al}$ 电极相比表现出更高的 $V_{\mathrm{oc}}, J_{\mathrm{sc}}$ 和 $\mathrm{FF}$, 器件性 能显著提高. 基于 ITO/ZnO/P59-60/P3HT:PCBM/PEDOT:PSS/Ag 反转型器件结构, Chen 等 ${ }^{[124]}$ 比较了阳极修 饰层侧链端基分别为磺酸钠及季铵盐离子的 P59 和 P60 时器件性能的变化, 研究发现, 器件性能表现出同样的 趋势, 光电转化效率分别提高至 $3.47 \%$ 和 $4.08 \%$. 


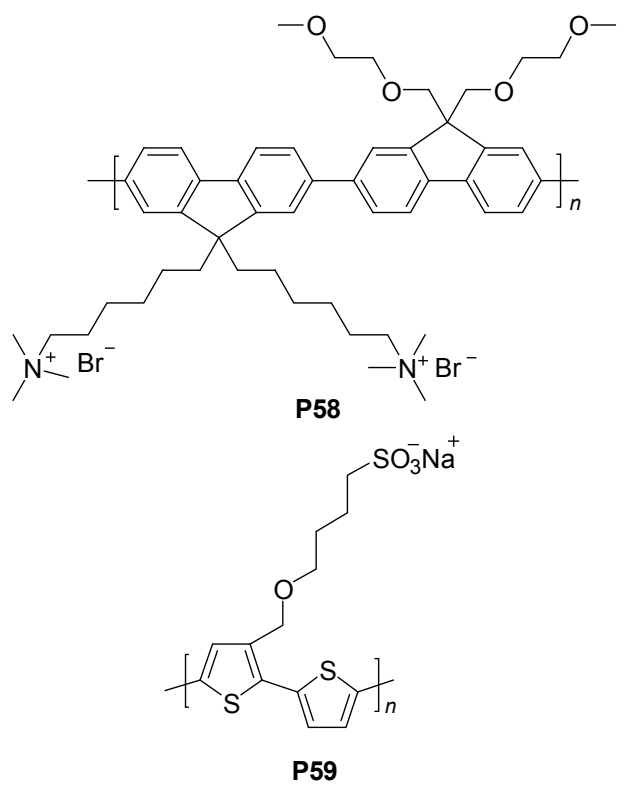

随后, 越来越多的研究开始围绕在聚电解质的结构 和性能关系之间 ${ }^{[126,127]}$. Bazan 等 ${ }^{[128]}$ 报道了基于 $\mathbf{P 6 0}$ 与 P61 的阴极修饰材料. 接触角、开尔文探针力显微镜以 及器件测试结果表明修饰材料可对混合膜形貌产生一 定影响. 且活性层与金属电极界面处特殊排列的修饰材 料还可形成界面偶极层, 有效提升载流子迁移与收集效 率, 具体表现在, 电极缓冲层(修饰层)的加入, 降低了 PCDTBT:PC71BM 活性层与电极的接触势垒, 短路电流 与器件效率均得到显著改善.

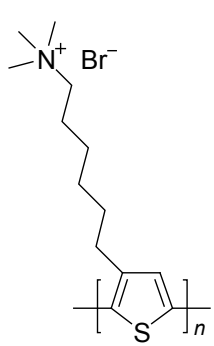

P60

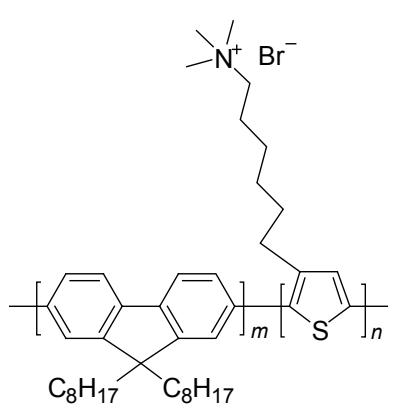

P61
随着分子结构设计调控手段的不断发展，越来越多 的新型高分子材料被用于器件的阴极修饰材料. 虽然不 同的活性层材料在阴极修饰后的表现各有差异, 但此类 课题的深入开展无疑对载流子传输、共混膜形貌优化以 及界面形态调控等问题的系统研究仍具有广泛意义.

\section{4 末端功能化基团型侧链}

理想的共轭聚合物光电材料应在可见光区具有宽 光谱和强吸收、与受体材料电子能级良好的能级匹配、 优异的载流子迁移性能、较好的成膜性和溶解性以及共 混液成膜过程中优异的自组装性能以形成纳米尺度相
分离的给体/受体互穿网络构 ${ }^{[136]}$. 为增强聚合物链间的 相互作用，提高聚合物材料的结晶性能，改善聚合物链 间的自组装性能，优化本体异质结共混膜形貌，近年来 不少研究工作者又对聚合物材料侧链端基加以功能化 基团修饰以改善器件光电性能的研究 ${ }^{[137,138]}$.

Bao 研究组 ${ }^{[139]}$ 出于改善材料溶解性以提高材料可 溶液加工性能的考虑, 在聚合物 P62 的结构基础上, 通 过对异靛蓝(Isoindigo)结构单元侧链末端加以键接可溶 性的硅氧烷制备出聚合物 P63. 光物理和电化学测试显 示, 聚合物的电子轨道能级以及禁带宽度并未发生明显 改变, 但结合 OFET 与 GIXD 测试结果, 相比 P62 $\left(\mu_{\mathrm{h}}=\right.$ $\left.0.30 \mathrm{~cm}^{2} \cdot \mathrm{V}^{-1} \cdot \mathrm{s}^{-1}\right)$, 鉴于 $\mathbf{P 6 3}$ 分子链间更小的 $\pi-\pi$ 堆叠距 离, 其空穴迁移率高达 $\mu_{\mathrm{h}}=2.00 \mathrm{~cm}^{2} \cdot \mathrm{V}^{-1} \cdot \mathrm{s}^{-1}$.

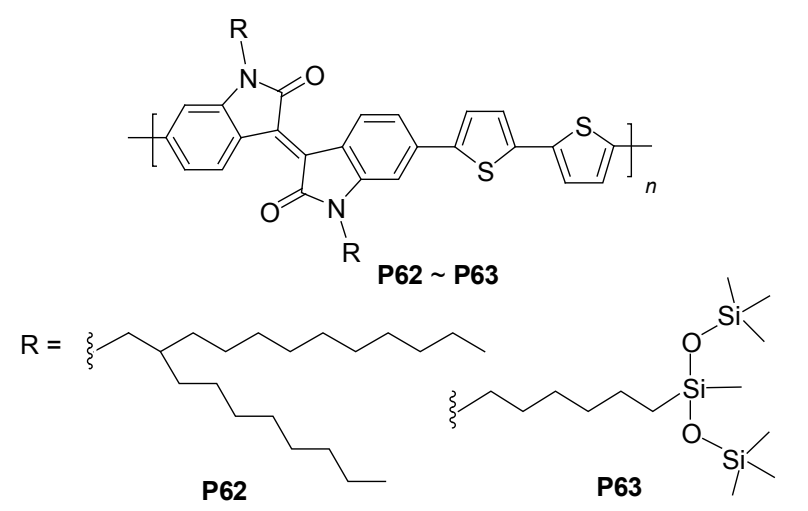

与此同时, 聚合物给体材料本身较低的混合商易导 致严重的相分离现象, 为改善聚合物材料与富勒烯衍生 物受体材料的相容性, 本实验室 ${ }^{[140]}$ 报道的基于二环戊 二烯苯并二噻吩(IDT)和双噻吩二酮吡咯并吡咯(DPP) 结构单元的 P64. 其结构特征表现在对受体单元 DPP 侧 链端基加以极性氧基基团修饰, 光物理及电化学测试结 果表明，聚合物经氰基基团修饰后电子轨道能级以及载 流子迁移率并未发生明显改变, 但表面能的提高则有效 的改善了聚合物给体材料与富勒烯衍生物受体材料的 相容性, 即聚合物的表面能越接 PC71BM 时, 与 PC71BM 相容性越优异, 混合层表面形貌越均匀, 相分 离越好. 氧基修饰的聚合物材料光电性能也有了很大的 提高, 基于 ITO/PEDOT:PSS/P64:PC71BM/Ca/Al 结构的 本体异质结器件转化效率提高至 $3.67 \%$.

Swager 等 ${ }^{[141]}$ 通过化学修饰将 P3HT 侧链端基引入 不同的芳香基团，成功制备出等规聚合物 $\mathbf{P 6 5} \sim \mathbf{P 7 0}$. 发现其以掺杂剂的形式加入 P3HT:PCBM 活性层中时, 可显著降低器件串联电阻, 提高短路电流, 改善光电效 率. 掺杂比例相当关键, 掺杂 $0.25 \mathrm{wt} \%$ 时, 载流子寿命 增加, 光电流得以提高, 但当掺杂剂含量大于 $5 \mathrm{wt} \%$, 则出现不利的相分离尺寸及串联电阻，器件效率反而下 降. 作者推测, 作为掺杂剂的 $\mathbf{P 6 5} \sim \mathbf{P 7 0}$ 可能位于混合 


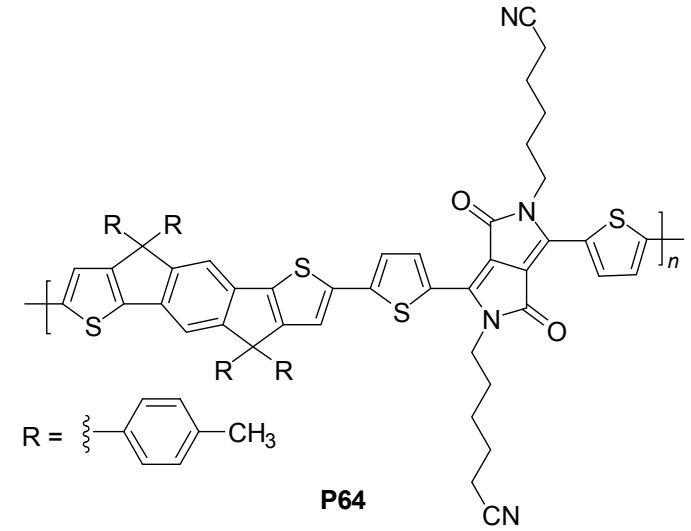

层中给-受体界面处, 且侧链端基的功能化基团可诱导 给-受体界面处产生偶极子, 提高混合膜中激子分离效 率, 降低复合几率. 诱导产生最大偶极子的 P70 可使得 器件效率在原来基础上提高近 $28 \%$.

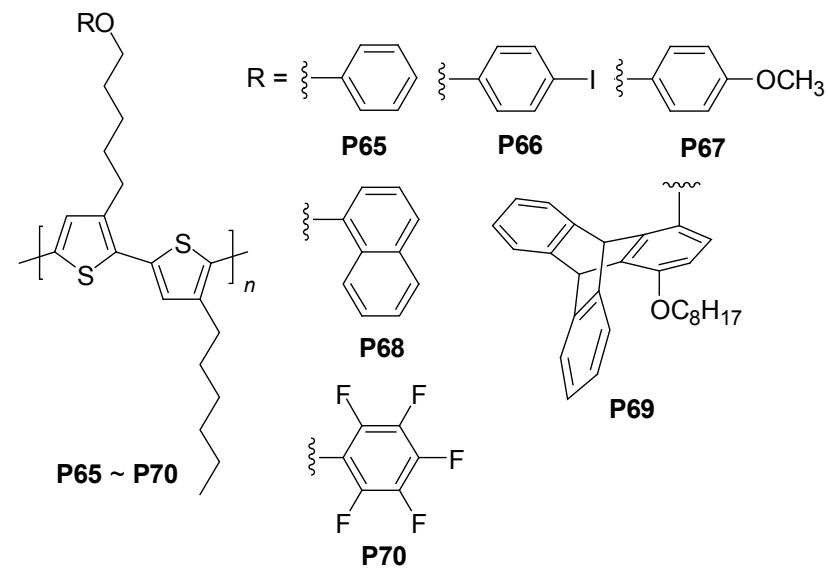

通过以上研究, 我们不难看出聚合物材料侧链端基 的功能化基团对光伏材料表面能、本体异质结膜形貌与 光电池效率具有重要影响. 这为今后聚合物光电材料分 子设计及光电器件性能改善提供了新思路和新方法.

\section{5 氟取代型}

近年来, 氟原子常被引入至共轭聚合物体系中以改 善聚合物材料的性能 ${ }^{[129 ~ 135]}$. You ${ }^{[131]}, \mathrm{Jen}^{[133]}$, Kleinhenz ${ }^{[134]}$, Yao 研究组 ${ }^{[135]}$ 等在此领域做了大量研究. 通过 比较, 他们发现氟吸电子基的引入使得聚合物材料的光 电效率得到很大提高, 他们认为性能的提高归因于: (1) 氟的强吸电子效应, 可降低聚合物的 HOMO 能级, 易于 获得较为理想的开路电压 $V_{\mathrm{oc}} ;(2)$ 分子间与分子内相互 作用易形成 $\mathrm{F}-\mathrm{H}$ 与 $\mathrm{F}-\mathrm{F}$ 键, 有利于链间的 $\pi-\pi$ 相互作 用, 更易于形成有益载流子传输的纳米级双连续膜结 构, 有效改善混合膜形貌.

此外, 由于较好热力学和化学稳定性以及良好的自 组装性能, 氟取代烷基型侧链亦可用于有机光电高分子

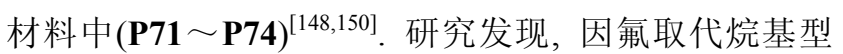

侧链自身良好的疏水性，使得其与烷基侧链同时引入聚 合物体系中时，烷基侧链与全氟或部分氟取代烷基侧链 二者之间会呈现相互独立的状态，易于材料自组装成高 度有序的纳米尺度结晶结构 ${ }^{[151,152]}$. 这一现象有望对调 控高分子链聚集及自组装性能方面的研究具有很好的 指导意义，但鉴于氟取代烷基在制备过程中的复杂性及 在有机溶剂中不佳的溶解性，使得近年来氟取代烷基型 侧链应用于 OPVs 与 OFETs 的报道研究相对较少.

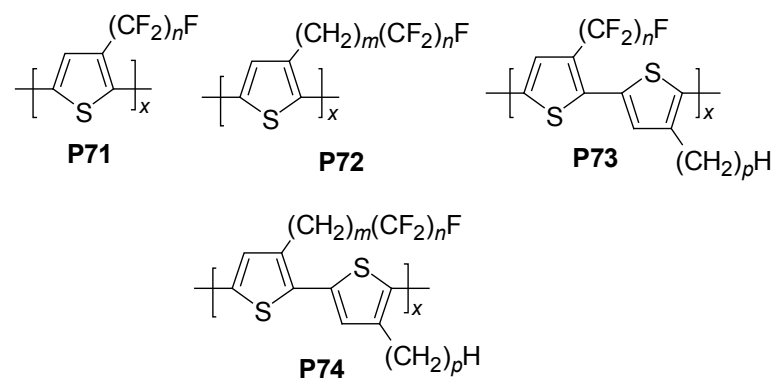

与此同时, 针对小分子共轭体系的侧链调控研究亦 具有重要的学术价值 ${ }^{[153 ~ 156]}$. 近些年来, 科学研究工作 者们在此领域做了大量的尝试与探索，发现小分子共轭 体系中引入的烷基型与混合型等侧链亦可改善材料的 光电特性 ${ }^{[153 \sim 156]}$. Gao 等 ${ }^{[153]}$ 发现, 烷基链长对一些共轭 有机小分子固态聚集行为和光电性质具有重要影响, 烷 基链可扮演着调控材料光电性质的 “功能基团” 作用. 以硫杂荎二酰亚胺(NDI2OD-DTYM2)为 $n$-型有机半导 体材料, 通过一系列的侧链修饰和优化, 电子迁移率可 达 $0.26 \mathrm{~cm}^{2} \cdot \mathrm{V}^{-1} \cdot \mathrm{s}^{-1}$. 随后, 在硫杂荎二酰亚胺 (NDI)的 结构基础上，他们又系统研究了烷基侧链的长度及支化 点对于材料光电性能的影响 ${ }^{[154]}$. 他们发现, 基于 NDI3HU-DTYM2 的 $n$-型有机半导体材料其电子迁移率 更是高达 $3.50 \mathrm{~cm}^{2} \cdot \mathrm{V}^{-1} \cdot \mathrm{s}^{-1}$.

\section{2 结论与展望}

综上所述, 聚合物材料侧链修饰对其吸收光

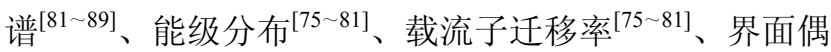

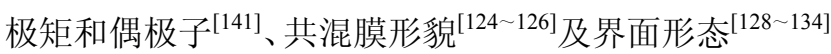
等方面会产生不同程度的影响. 对材料吸收光谱和载流 子迁移率的影响具体表现在侧链可有效影响聚合物链 的排列形态，导致二面角发生变化，进而影响分子平面 性、主链有效共轭程度及聚合物链间 $\pi-\pi$ 相互作 用 ${ }^{[142 ~ 147]}$. 对能级分布的影响则主要是侧链给拉电子效 应的作用. 而活性层膜形貌的改变则主要是由于侧链修 饰后聚合物分子链的聚集性和结晶性、材料表面能及与 受体材料的相容性发生了不同程度的变化. 与此同时, 对侧链调控还可优化活性层与金属电极的接触界面, 减 小器件的并、串联电阻; 且自身在活性层与金属电极界 
面处的特殊排列还可形成特定取向的偶极子，因此，为 界面形态、界面偶极子和偶极矩的调控研究提供了思路.

尽管目前侧链调控在有机光电高分子材料改性研 究中有着较为广泛的研究与应用, 但仍存在尚未解决的 难题, 急需今后的研究者们开展更为细致及深入的工 作：(1)借助于先进的理论模拟计算, 预测侧链修饰特别 是共轭型侧链对于聚合物材料的吸收光谱、载流子迁移 率、能级分布等方面的影响; (2)吸电子型侧链的引入虽 可有效降低聚合物 HOMO 与 LUMO 的轨道能量, 改善 器件的开路电压 $V_{\mathrm{oc}}$, 但相关研究却很少涉及其对共混 膜形貌的影响; (3)水/醇溶性侧链修饰后聚合物材料光 电性能得到改善的内在机制亦有待进一步探索和验证; (4)通过侧链端基调控界面偶极矩, 促进电荷分离和减 少电荷复合, 对提高光电性能具有重要的意义, 还亟需 对此进行更为深入的研究和探索.

因此, 我们有理由相信, 随着有机光电材料分子结 构的不断优化以及器件制备与加工工艺的不断改善, 侧 链调控必然带动诸如有机场效应晶体管、有机发光二极 管以及有机太阳能电池等有机电子领域的飞速发展, 有 望引发新一轮的技术变革.

\section{References}

[1] Wang, Y.; Liu, M.; Chen, S. J.; Peng, R. X.; Ge, Z. Y. Chem. Mater. 2013, 25, 3196.

[2] Liu, Z.; Xu, F.; Yan, D. T. Acta Chim. Sinica 2014, 72, 171 (in Chinese). (刘震，徐丰，严大东，化学学报, 2014, 72, 171.)

[3] Ma, Z. F.; Wang, E. G.; Jarvid, M. E.; Henriksson, P.; Inganas, O.; Zhang, F. L.; Andersson, M. R. J. Mater. Chem. 2012, 22, 2306.

[4] Dou, L. T.; Gao, J.; Richard, E.; You, J. B.; Chen, C. C.; Cha, K. C.; He, Y. J.; Li, G.; Yang, Y. J. Am. Chem. Soc. 2012, 134, 10071.

[5] Zhang, S. Q.; Ye, L.; Wang, Q.; Li, Z. J.; Guo, X.; Huo, L. J.; Fan, H. L.; Hou, J. H. J. Phys. Chem. C 2013, 117, 9550.

[6] Higashihara, T.; Wu, H. C.; Mizobe, T.; Lu, C. E.; Ueda, M.; Chen, W. C. Macromolecules 2012, 45, 904.

[7] Intemann, J. J.; Yao, K.; Yip, H. L.; Xu, Y. X.; Li, Y. X.; Liang, P. W.; Ding, F. Z.; Li, X. S.; Jen, A. K. Y. Chem. Mater. 2013, 25, 3188 .

[8] Wang, M.; Hu, X. W.; Liu, P.; Li, W.; Gong, X.; Huang, F.; Cao, Y. J. Am. Chem. Soc. 2011, 133, 9638

[9] Bedi, A.; Senanayak, S. P.; Narayan, K. S.; Zade, S. S. Macromolecules 2013, 46, 5943.

[10] Cui, W. B.; Wudl, F. Macromolecules 2013, 46, 7232.

[11] Li, H.; Liu, F. B.; Wang, X. D.; Gu, C. L.; Wang, P.; Fu, H. B. Macromolecules 2013, 46, 9211.

[12] Zhou, E. J.; Cong, J. Z.; Hashimoto, K.; Tajima, K. Macromolecules 2013, 46, 763 .

[13] Lu, S. F.; Drees, M.; Yao, Y.; Boudinet, D.; Yan, H.; Pan, H. L.; Wang, J. Q.; Li, Y. N.; Usta, H.; Facchetti, A. Macromolecules 2013, 46, 3895.

[14] Siram, R. B. K. S.; Smith, J.; Anthopoulos, T. D.; Patil, S. J. Mater. Chem. 2012, 22, 4450.

[15] Yang, R. Q.; Wu, H. B.; Cao, Y.; Bazan, G. C. J. Am. Chem. Soc. 2006, 45, 14422.
[16] Chen, H. C.; Chen, Y. H.; Liu, C. C.; Chien, Y. C.; Chou, S. W.; Chou, P. T. Chem. Mater. 2012, 24, 4766.

[17] Wang, N.; Chen, Z.; Wei, W.; Jiang, Z. H. J. Am. Chem. Soc. 2013, 135,17060 .

[18] Yamamoto, S.; Ohkita, H.; Benten, H.; Ito, S.; Yamamoto, S.; Kitazawa, D.; Tsukamoto, J. J. Phys. Chem. C 2013, 117, 11514

[19] Umeyama, T.; Watanabe, Y.; Douvogianni, E.; Imahori, H. J. Phys. Chem. C 2013, 117, 21148.

[20] Jin, E.; Du, C.; Wang, M.; Li, W. W.; Li, C. H.; Wei, H. D.; Bo, Z. S. Macromolecules 2012, 45, 7843 .

[21] Li, J.; Zhao, Y.; Tan, H. S.; Guo, Y.; Di, C. A.; Yu, G.; Liu, Y.; Lin, M.; Lim, S. H.; Zhou, Y.; Su, H.; Ong, B. S. Sci. Rep. 2012, 2, 754.

[22] Lei, T.; Dou, J. H.; Ma, Z. J.; Yao, C. H.; Liu, C. J.; Wang, J. Y.; Pei, J. J. Am. Chem. Soc. 2012, 134, 20025.

[23] An, C. B.; Puniredd, S. R.; Guo, X.; Stelzig, T; Zhao, Y. F.; Pisula, W.; Baumgarten, M. Macromolecules 2014, 47, 979.

[24] Shi, S. W.; Xie, X. D.; Gao, C.; Shi, K. L.; Chen, S.; Yu, G.; Guo, L. H.; Li, X. Y.; Wang, H. Q. Macromolecules 2014, 47, 616.

[25] Perez, L. A.; Zalar, P.; Ying, L.; Schmidt, K.; Toney, M. K.; Nguyen, T. Q.; Bazan, G. C.; Kramer, E. J. Macromolecules 2014, 47, 1403.

[26] Wan, M. X.; Zhu, H. B.; Deng, H. Q.; Jin, L.; Guo, J.; Huang, Y. L. Polym. Chem. 2013, 51, 3477.

[27] Zhao, Z.; Zhang, F. J.; Zhang, X.; Yang, X. D.; Li, H. X.; Gao, X. K.; Di, C. A.; Zhu, D. B. Macromolecules 2013, 46, 7705.

[28] Wu, Y.; Jing, Y.; Guo, X.; Zhang, S. Q.; Zhang, M. J.; Huo, L. J.; Hou, J. H. Polym. Chem. 2013, 4, 536.

[29] Mei, J. G.; Bao, Z. N. Chem. Mater. 2014, 1, 604.

[30] Wang, Y.; Xin, X.; Lu, Y.; Xiao, T.; Xu, X. F.; Zhao, N.; Hu, X.; Ong, B. S.; Ng, S. C. Macromolecules 2013, 24, 9587.

[31] Hou, J. H.; Chen, H. Y.; Zhang, S. Q.; Chen, R. I.; Yang, Y.; Wu, Y.; Li, G. J. Am. Chem. Soc. 2009, 131, 15586

[32] Verswyvel, M.; Koeckelberghs, G. Polym. Chem. 2012, 3, 3203.

[33] Kola, S.; Kim, J. H.; Ireland, R.; Yeh, M. L.; Smith, K.; Guo, W. M.; Katz, H. E. ACS Macro Lett. 2013, 2, 664.

[34] Xin, H.; Kim, F. S.; Jenekhe, S. A. J. Am. Chem. Soc. 2008, 130, 5424.

[35] Szarko, J. M.; Guo, J.; Liang, Y.; Lee, B.; Rolczynski, B. S.; Strzalka, J.; Xu, T.; Loser, S.; Marks, T. J.; Yu, L.; Chen, L. X. Adv. Mater. 2010, 22, 5468.

[36] Lei, T.; Dou, J. H.; Pei, J. Adv. Mater. 2012, 24, 6457.

[37] Wang, C.; Dong, H.; Hu, W.; Liu, Y.; Zhu, D. Chem. Rev. 2011, $112,2208$.

[38] Qin, R.; Li, W.; Li, C.; Du, C.; Veit, C.; Schleiermacher, H. F.; Andersson, M.; Bo, Z.; Liu, Z.; Inganäs, O.; Wuerfel, U.; Zhang, F. J. Am. Chem. Soc. 2009, 131, 14612

[39] Matthews, J. R.; Niu, W.; Tandia, A.; Wallace, A. L.; Hu, J.; Lee, W. Y.; Giri, G.; Mannsfeld, S. C. B.; Xie, Y.; Cai, S.; Fong, H. H.; Bao, Z.; He, M. Chem. Mater. 2013, 25, 782.

[40] Li, Y.; Sun, B.; Sonar, P.; Singh, S. P. Org. Electron. 2012, 13, 1606.

[41] Akkerman, H. B.; Mannsfeld, S. C. B.; Kaushik, A. P.; Verploegen, E.; Burnier, L.; Zoombelt, A. P.; Saathoff, J. D.; Hong, S.; Atahan, E. S.; Liu, X.; Aspuru, G. A.; Toney, M. F.; Clancy, P.; Bao, Z. J. Am. Chem. Soc. 2013, 135, 11006.

[42] Shi, Q. Q.; Fan, H. J.; Liu, Y.; Chen, J. M.; Ma, L. C.; Hu, W. P.; Shuai, Z. G.; Li, Y. F.; Zhan, X. W. Macromolecules 2011, 44, 4230.

[43] Lu, K.; Fang, J.; Yan, H.; Zhu, X. W.; Yi, Y. P.; Wei, Z. X. Org. Electron. 2013, 14, 2652

[44] Deng, Y. F.; Chen, Y .G.; Liu, J.; Liu, L. H.; Tian, H. K.; Xie, Z. 
Y.; Geng, Y. H.; Wang, F. S. ACS Appl. Mater. Interfaces 2013, 5, 5741.

[45] Ma, Z. F.; Wang, E. G.; Jarvid, M. E.; Henriksson, P.; Inganas, O.; Zhang, F. L.; Andersson, M. R. J. Mater. Chem. 2012, 22, 2306.

[46] Li, Y. X.; Zou, J. Y.; Yip, H. L.; Li, C. Z.; Zhang, Y.; Chueh, C. C.; Intemann, J.; Xu, Y. X.; Liang, P. W.; Chen, Y.; Jen, A. K. Y. Macromolecules 2013, 46, 5497.

[47] Huang, Y. C.; Lu, T. C.; Huang, C. I. Polymer 2013, 54, 6489.

[48] Lee, S. K.; Cho, S.; Tong, M. H.; Seo, J. H.; Heeger, A. J. Polym. Chem. 2011, 49, 1821

[49] Price, S. C.; Stuart, A. C.; You, W. Macromolecules 2010, 43, 4609.

[50] Huang, H.; Youn, J.; Ponce Ortiz, R.; Zheng, Y.; Facchetti, A.; Marks, T. Chem. Mater. 2011, 23, 2185.

[51] Kang, I.; Yun, H. J.; Chung, D. S.; Kwon, S. K.; Kim, Y. H. J. Am. Chem. Soc. 2013, 135, 14896.

[52] Okamoto, T.; Bao, Z. J. Am. Chem. Soc. 2007, 129, 10308.

[53] Shi, C.; Yao, Y.; Yang, Y.; Pei, Q. J. Am. Chem. Soc. 2006, 128, 8980.

[54] Li, W. W.; Hendriks, K. H.; Furlan, A.; Roelofs, W. S. C.; Wienk, M. M.; Janssen, R.J. J. Am. Chem. Soc. 2013, 50, 18942.

[55] Yamamoto, T.; Ikai, T.; Kuzuba, M.; Kuwabara, T.; Maeda, K.; Takahashi, K.; Kanoh, S. Macromolecules 2011, 44, 6659.

[56] Zhou, H. X.; Yang, L. Q.; Stoneking, S.; You, W. ACS Appl. Mater. Interfaces 2010, 5, 1377.

[57] Zhou, E. J.; Cong, J. Z.; Hashimoto, K.; Tajima, K. Macromolecules 2013, 46, 763.

[58] Hou, J. H.; Chen, H. S.; Zhang, S. Q.; Yang, Y. J. Phys. Chem. C 2009, 113, 21202.

[59] Wang, E. G.; Hou, L. T.; Wang, Z. Q.; Ma, Z. F.; Hellstrom, S.; Zhuang, W. L.; Zhang, F. L.; Inganas, O.; Andersson, M. R. Macromolecules 2011, 44, 2067.

[60] Jager, M.; Trattnig, R.; Postl, M.; Haas, W.; Kunert, B.; Resel, R.; Hofer, F.; Klug, A.; Trimmel, G.; List, E. J. W. Polym. Phys. 2013, 51,1400

[61] Qin, R. P.; Jiang, Y. R.; Ma, H.; Yang, L.; Liu, H. Z.; Chang, F. G. J. Appl. Polym. Sci. 2013, 5, 2671.

[62] Qin, R. P.; Li, W. W.; Li, C. H.; Du, C.; Veit, C.; Schleiermacher, H. F.; Andersson, M.; Bo, Z. S.; Liu, Z. P.; Inganas, O.; Wuerfel, O.; Zhang, F. L. J. Am. Chem. Soc. 2009, 41, 14612.

[63] Zhang, J.; Cai, W. Z.; Huang, F.; Wang, E. G.; Zhong, C. M.; Liu, S. J.; Wang, M.; Duan, C. H.; Yang, T. B.; Cao, Y. Macromolecules 2011, 44, 894.

[64] Chen, H. C.; Chen, Y. H.; Liu, C. C.; Chien, Y. C.; Chou, S. W.; Chou, P. T. Chem. Mater. 2012, 24, 4766.

[65] Yamamoto, S.; Ohkita, H.; Benten, H.; Ito, S.; Yamamoto, S.; Kitazawa, D.; Tsukamoto, J. J. Phys. Chem. C 2013, 117, 11514.

[66] Chang, H. H.; Tsai, C. E.; Lai, Y. Y.; Liang, W. W.; Hsu, S. L.; Hsu, C. S.; Cheng, Y. J. Macromolecules 2013, 46, 7715.

[67] Huang, Y.; Zhang, M. Q.; Ye, L.; Guo, X.; Han, C. C.; Li, Y. F.; Hou, J. H. J. Mater. Chem. 2012, 22, 5700

[68] Seri, M.; Bolognesi, M.; Chen, Z. H.; Lu, S. F.; Koopman, W.; Facchetti, A.; Muccini, M. Macromolecules 2013, 46, 6419.

[69] Zhang, C.; Sun, J. Y.; Li, R.; Sun, S. S. J.; Lafalce, E.; Jiang, X. M. Macromolecules 2011, 16, 6389

[70] Speros, J. C.; Martinez, H.; Paulsen, B. D.; White, S. P.; Bonifas, A. D.; Goff, P. C.; Frisbie, C. D.; Hillmyer, M. A. Macromolecules 2013, 13, 5184 .

[71] Nguyen, L. H.; Neugebauer, H.; Sariciftci, N. S. Thin Solid Films 2008, 516, 3978.

[72] Genoe, J.; Poortmans, J.; Heremans, P. Org. Electron. 2008, 9, 7401 .
[73] Hwang, I. W.; Xu, Q. H.; Soci, C.; Chen, B. Q.; Jen, A. K. Y.; Moses, D.; Heeger, A. J. Adv. Funct. Mater. 2007, 17, 563.

[74] Huo, L. J.; Teresa, L.; Chen, T. L.; Zhou, Y.; Hou, J. H.; Chen, H. Y.;Yang,Y.; Li, Y. F. Macromolecules 2009, 42, 4377.

[75] Liang, Y. Y.; Wu,Y.; Feng, D. Q.; Tsai, S. T.; Son, H. J.; Li, G.; Yu, L. P. J. Am. Chem. Soc. 2009, 131, 56.

[76] Hou, J. H.; Chen, H. Y.; Zhang, S. Q.; Chen, R. I.; Yang, Y.; Wu, Y.; Li, G. J. Am. Chem. Soc. 2009, 131, 15586.

[77] Facchetti, A. Chem. Mater. 2011, 23, 733.

[78] Hwang, I. W.; Kim, J. Y.; Cho, S.; Yuen, J.; Coates, N.; Lee, K.; Heeney, M.; Mcculloch, I.; Moses, D.; Heeger, A. J. J. Phys. Chem. C 2008, 112, 7853 .

[79] Feng, J.; Zhang, Q.; Li, W.; Li, Y.; Yang, M.; Cao, Y. J. Appl. Polym. Sci. 2008, 109, 22831.

[80] Wienk, M. M.; Turbiez, M.; Gilot, J.; Janseen, R. A. J. Adv. Mater. 2008, 20, 2556.

[81] Yuan, M. J.; Durban, M. M.; Kazarinoff, P. D.; Zeigler, D. F.; Rice, A. H.; Segawa, Y.; Luscombe, C. K. Polym Chem. 2013, 51, 4061.

[82] Mei, C. Y.; Liang, L.; Zhao, F. G.; Wang, J. T.; Yu, L. F.; Li, Y. X.; Li, W. S. Macromolecules 2013, 46, 7920.

[83] Sakthivel, P.; Song, H. S.; Chakravarthi, N.; Lee, J. W. L.; Gal, Y. S.; Hwang, S.; Jin, S. H. Polymer 2014, 54, 4883.

[84] Kim, J. H.; Song, C. E.; Kim, H. E.; Kang, I. N.; Shin, W. S.; Park, M. J.; Hwang, D. H. Polym. Chem. 2013, 51, 4136.

[85] Zhang, J.; Cai, W. Z.; Huang, F.; Wang, E. G.; Zhong, C. M.; Liu, S. J.; Wang, M.; Duan, C. H.; Yang, T. B.; Cao, Y. Macromolecules 2011, 44, 894.

[86] Cho, H. H.; Kang, T. K.; Kim, K. H.; Kang, H.; Kim, H. J.; Kim, B. J. Macromolecules 2012, 45, 6415.

[87] Ong, K. H.; Lim, S. L.; Tan, H. S.; Wong, H. K.; Li, J.; Ma, Z.; Moh, L. C. H.; Lim, S. H.; Mello, J. C.; Chen, Z. K. Adv. Mater. 2011, 23, 1409.

[88] Huo, L. J.; Ye, L.; Wu, Y.; Li, Z. J.; Guo, X.; Zhang, M. J.; Zhang, S. Q.; Hou, J. H. Macromolecules 2012, 45, 6923.

[89] Zhang, A. Q.; Ye, L.; Wang, Q.; Li, Z. J.; Guo, X.; Huo, L. J.; Fan, H. L.; Hou, J. H. J. Phys. Chem. C 2013, 117, 9550.

[90] Yun, H. J.; Lee, Y. J.; Yoo, S. J.; Chung, S. S.; Kim, Y. H.; Kwon, S. K. Chem. Eur. J. 2013, 19, 13242.

[91] Chen, C. P.; Hsu, H. L. Macromol. Rapid Commun. 2013, 34, 1623.

[92] Ong, B. S.; Wu, Y.; Liu, P.; Gardner, S. J. Am. Chem. Soc. 2004, 126,3378

[93] Giacalone, F.; Segura, J. L.; Martin, N.; Catellani, M.; Luzzati, S.; Lupsac, N. Org. Lett. 2003, 5, 16691.

[94] Li, G.; Shrotriya, V.; Huang, J. S.; Yao, Y.; Moriarty, T.; Emery, K.; Yang, Y. Nat. Mater. 2005, 4, 864.

[95] Li, Y. Acc. Chem. Res. 2012, 45, 723.

[96] Min, J.; Zhang, Z. G.; Zhang, S.; Li, Y. Chem. Mater. 2012, 24, 3247.

[97] Zhan, X. W.; Tan, Z. A.; Domercq, B.; An, Z.; Zhang, X.; Barlow, S.; Li, Y. F.; Zhu, D. B.; Kippelen, B.; Marder, S. R. J. Am Chem Soc. 2007, 129, 7264.

[98] Hou, J. H.; Huo, L. J.; He, C.; Yang, C. H.; Li, Y. F. Macromolecules 2006, 39: 594.

[99] Hou, J. H.; Tan, Z. A.; Yan, Y.; He, Y. J.; Yang, C. H.; Li, Y. F. J. Am. Chem. Soc. 2006, 128, 4911.

[100] Huang, F.; Chen, K. S.; Yip, H. L.; Hau, S. K.; Acton, O.; Zhang, Y.; Luo, J. D.; Jen, A. K. Y. J. Am. Chem. Soc. 2009, 39, 13886.

[101] Singh, S. P.; Kumar, C. P.; Nagarjuna, P.; Sharma, G. D.; Biswas, S.; Mikroyannidis, J. A. J. Phys. Chem. C 2013, 117, 13350

[102] Duan. C. H.; Chen, K. S.; Yip, H. L.; Liu, S. J.; Zhang, Z.; Jen, A. K. Y.; Cao, Y. Chem. Mater. 2010, 23, 6444.

[103] Zhang, Z. G.; Liu, Y. L.; Yang, Y.; Hou, K. Y.; Peng, B.; Zhao, G. 
J.; Guo, X.; Kang, E. T.; Li, Y. F. Macromolecules 2010, 22, 9376.

[104] Gu, Z. J.; Tang, P.; Zhao, B.; Luo, H.; Guo, X.; Chen, H. J.; Yu, G.; Liu, X. P.; Shen, P.; Tan, S. T. Macromolecules 2012, 45, 2359.

[105] Kang, H. Y.; Zhao, B.; Cao, Z. C.; Zhong, J.; Li, H. H.; Pei, Y.; Shen, P.; Tan, S. T. Eur. Polym. J. 2013, 49, 2738.

[106] Tan, H.; Deng, X. P.; Yu, J. T.; Zhao, B. F.; Wang, Y. F.; Liu, Y.; Zhu, W. G.; Wu, H. B.; Cao, Y. Macromolecules 2013, 46, 113.

[107] Wang, C. W.; Zhao, B.; Cao, Z. C.; Shen, P.; Tan, Z.; Li, Z. L.; Tan, S. T. Chem. Commun. 2013, 49, 3857.

[108] Zhou, H. X.; Yang, L. Q.; You, W. Macromolecules 2012, 45, 607.

[109] Yao, K.; Chen, L.; Chen, Y.; Li, F.; Wang, P. J. Mater. Chem. 2011, 21, 13780.

[110] Yang, X.; Loos, J.; Veenstra, S. C.; Verhees, W. J. H.; Wienk, M. M. N. Nano Lett. 2005, 5, 579.

[111] Scherf, U.; Gutacker, A.; Koenen, N. Acc. Chem. Res. 2008, 41, 1086.

[112] Chen, X. L.; Bao, Z.; Schon, J. H.; Lovinger, A. J.; Lin, Y. Y.; Crone, B.; Dodabalapur, A.; Batlogg, B. Appl. Phys. Lett. 2001, 78, 228.

[113] Garcia, A.; Brzezinski, J. Z.; Nguyen, T. Q. J. Phys. Chem C 2009, $113,2950$.

[114] Kang, M.; Nag, O. K.; Nayak, R. R.; Hwang, S.; Suh, H.; Woo, H. Y. Macromolecules 2009, 42, 2708.

[115] Xu, X. F.; Han, B.; Chen, J. W.; Peng, J. B.; Wu, H. B.; Cao, Y. Macromolecules 2011, 44, 4204.

[116] Brabec, C. J.; Cravino, A.; Meissner, D.; Sariciftci, N.S.; Fromherz, T.; Rispens, M. T.; Sanchez, L.; Hummelen, J. C. Adv. Funct. Mater. 2001, 11, 374

[117] Yang, T.; Wang, M.; Duan, C.; Hu, X.; Huang, L.; Peng, J.; Huang, F.; Gong, X. Energy Environ. Sci. 2012, 5, 8208.

[118] Yang, R. Q.; Wu, H. B.; Cao, Y.; Bazan, G. C. J. Am. Chem. Soc. 2006, 128, 14422.

[119] Zhao, Y.; Xie, Z. Y.; Qin, C. J.; Qu, Y.; Geng, Y. H.; Wang, L. X. Sol. Energy Mater. Sol. Cells 2009, 93, 604.

[120] Shao, M.; He, Y. J.; Hong, K. L.; Rouleau, C. M.; Geohegan, D. B.; Xiao, K. Polym. Chem. 2013, 4, 5270.

[121] Kanimozhi, C.; Gross, N. Y.; Chou, K. W.; Amassian, A.; Anthopoulos, T. D.; Patil, S. J. Am. Chem. Soc. 2012, 134, 16532.

[122] Lee, E.; Hammer, B.; Kim, J. K.; Page, Z.; Emrick, T.; Hayward, R. C. J. Am. Chem. Soc. 2011, 133, 10390.

[123] Sun, J. M.; Zhu, Y. X.; Xu, X. F.; Lan, L. F.; Zhang, L. J.; Cai, P.; Chen, J. W.; Peng, J. B.; Cao, Y. J. Phys. Chem. C 2012, 116, 1418.

[124] Xie, C.; Chen, L.; Chen, Y. W. J. Phys. Chem. C 2013, 117, 24804.

[125] Na, S. I.; Oh, S. H.; Kim, S. S.; Kim, D. Y. Org. Electron. 2009, $10,496$.

[126] Yang, J.; Zhu, R.; Hong, Z. R.; He, Y. J.; Kumar, A.; Li, Y. F.; Yang, Y. Adv. Mater. 2011, 23, 346.

[127] Yao, K.; Chen, L.; Chen, Y.; Li, F.; Wang, P. J. Mater. Chem. 2011, 21, 13780.

[128] Seo, J. H.; Gutacker, A.; Sun, Y. M.; Wu, H. B.; Huang, F.; Cao, Y.; Scherf, U.; Heeger, A. J.; Bazan, G. C. J. Am. Chem. Soc. 2011, 133, 8416.

[129] Yang, Y. C.; Wu, R. M.; Wang, X.; Xu, X. P.; Li, Z. J.; Li, K.; Peng, Q. Chem. Commun. 2014, 50, 439.

[130] Geng, Y.; Wei, Q.; Hashimoto, K.; Tajima, K. Chem. Mater. 2011, 23, 42571.

[131] Price, S. C.; Stuart, A. C.; Yang, L. Q.; Zhou, H. X.; You, W. J. Am. Chem. Soc. 2011, 133, 4625.
[132] Zhang, Y.; Chien, S. C.; Chen, K. S.; Yip, H. L.; Sun, Y.; Davies, J. A.; Chen, F. C.; Jen, A. K.Y. Chem. Commun. 2011, 47, 11026.

[133] Zhang, Y.; Zou, J. Y.; Cheuh, C. C.; Yip, H. L.; Jen, A. K. Y. Macromolecules 2012, 45, 5427.

[134] Kleinhenz, N.; Yang, L. Q.; Zhou, H. X.; Price, S. C.; You, W. Macromolecules 2011, 44, 872.

[135] Yao, K.; Chen, L.; Chen, X.; Chen, Y. W. Chem. Mater. 2013, 25, 897.

[136] Noriega, R.; Rivnay, J.; Vandewal, K.; Koch, F. P. V.; Stingelin, N.; Smith, P.; Toney, M. F.; Salleo, A. Nat. Mater. 2013, 12, 1038.

[137] Duan, C. H.; Cai, W. Z.; Hsu, B. B. Y.; Zhong, C. M.; Zhang, K.; Liu, C. C.; Hu, Z. C.; Huang, F.; Bazan, G. C.; Heeger, A. J.; Cao, Y. Energy Environ. Sci. 2013, 6, 3022.

[138] Yao, K.; Chen, L.; Li, F.; Wang, P. S.; Chen, Y. W. J. Phys. Chem. C 2012, 116, 714 .

[139] Mei, J. G.; Kim, D. H.; Ayzner, A. L.; Toney, M. F.; Bao, Z. N. J. Am. Chem. Soc. 2011, 133, 20130.

[140] Sun, Y.; Chien, S. C.; Yip, H. L.; Chen, K. S.; Zhang, Y.; Davies, J. A.; Chen, F. C.; Lin, B.; Jen, A. K.Y. J. Mater. Chem. 2012, 22, 5587.

[141] Lobez, J. M.; Andrew, T. L.; Bulovic, V.; Swager, T. M. ACS Nano 2012, 6, 3044.

[142] Fang, L.; Zhou, Y.; Yao, Y. X.; Diao, Y.; Lee, W. Y.; Appleton, A. L.; Allen, R.; Reinspach, J.; Mannsfeld, S. C. B.; Bao, Z. N. Chem. Mater. 2013, 25, 4874.

[143] Lipomi, D. J.; Tee, B. C. K.; Vosgueritchian, M.; Bao, Z. Adv. Mater. 2011, 23, 1771.

[144] Li, X. F.; Zhao, B.; Cao, Z. C.; Shen, P.; Tan, S. T. Acta Chim. Sinica 2012, 70, 2043 (in Chinese). (李新炜，赵斌，曹镇财，沈平，谭松庭，化学学报，2012，70, 2043.)

[145] Zou, W. B.; Liu, Y.; Jia, Q. M.; Ge, Z. Y. Chin. J. Org. Chem. 2013, 33, 1522 (in Chinese). (邹文武, 刘颖, 贾庆明, 葛子义, 有机化学, 2013, 33, 1522.)

[146] He, P.; Li, Z. F.; Hou, Q. F.; Wang, Y. L. Chin. J. Org. Chem. 2013, 33, 288 (in Chinese).

(和平, 李在房, 侯秋飞, 王艳玲, 有机化学, 2013, 33, 288.)

[147] You, J.; Dou, L.; Yoshimura, K.; Kato, T.; Ohya, K.; Moriarty, T.; Emery, K.; Chen, C. C.; Gao, J.; Li, G.; Yang, Y. Nat. Commun. 2013, 4, 1446.

[148] Wang, B.; Watt, S.; Hong, M.; Domercq, B.; Sun, R.; Kippelen, B.; Collard, D. M. Macromolecules 2008, 41, 5156.

[149] Li, L.; Counts, K. E.; Kurosawa, S.; Teja, A. S.; Collard, D. M. Adv. Mater. 2004, 16, 180

[150] Li, L.; Collard, D. M. Macromolecules 2004, 38, 372.

[151] Yao, K.; Chen, L.; Chen, X.; Chen, Y. Chem. Mater. 2013, 25, 897.

[152] Geng, Y.; Wei, Q.; Hashimoto, K.; Tajima, K. Chem. Mater. 2011, $23,4257$.

[153] Hu, Y. B.; Gao, X. K.; Di, C. A.; Yang, X. D.; Zhang, F.; Liu, Y. Q.; Li, H. X.; Zhu, D. B. Chem. Mater. 2011, 23, 1204.

[154] Zhang, F. J.; Hu, Y. B.; Schuettfort, T.; Di, C. A.; Gao, X. K.; McNeill, C. R.; Thomsen, L.; Mannsfeld, S. C. B.; Yuan, W.; Sirringhaus, H.; Zhu, D. B. J. Am. Chem. Soc. 2013, 135, 2338.

[155] Fu, L.; Fu, W. H.; Cheng, P.; Xie, Z. X.; Fan, C. C.; Shi, M. M.; Ling, J.; Hou, J. H.; Zhan, X. W.; Chen, H. Z. J. Mater. Chem. A 2014, 2, 6589 .

[156] Chen, P.; Ye, L.; Zhao, X. G.; Hou, J. H.; Li, Y. F.; Zhan, X. W. Energy Environ. Sci. 2014, 7, 1351. 